\title{
Yield from genetic variability of bread wheat (Triticum aestivum L.) genotypes under water stress condition: A case study of Tandojam, Sindh
}

\author{
Ayaz Latif Siyal ${ }^{1 *}$, Fozia Khan Siyal ${ }^{2}$ and Tahira Jatt ${ }^{2}$ \\ 1. Department of Plant Breeding \& Genetics, Sindh Agriculture University Tandojam, Sindh-Pakistan \\ 2. Department of Botany Shah Abdul Latif University Khairpur (Mirs) Sindh-Pakistan \\ *Corresponding author's email: latifayazsiyal@gmail.com

\section{Citation}

Ayaz Latif Siyal, Fozia Khan Siyal and Tahira Jatt. Yield from genetic variability of bread wheat (Triticum aestivum L.) genotypes under water stress condition: A case study of Tandojam, Sindh. Pure and Applied Biology. Vol. 10, Issue 3, pp841-860. http://dx.doi.org/10.19045/bspab.2021.100087

\begin{tabular}{llll}
\hline \hline Received: 01/10/2020 & Revised: 01/12/2020 & Accepted: 09/12/2020 & Online First: 14/12/2020 \\
\hline \hline
\end{tabular}

\section{Abstract}

Water stress considered as a major abiotic factor limiting plant growth and affecting productivity is a serious global problem of Agriculture. To determine the effects of drought stress on various yield and yield associated traits of bread wheat. Fifteen newly evolved genotypes and two check varieties viz., NIA-Amber (drought-sensitive), Khirman (drought-tolerant) were selected for various water stress under field observations. Experiment was conducted in split plot design using three treatments viz., single irrigation, two irrigations three irrigations. The test entries comprised of 11 exotic wheat genotypes (BWDYT-1 to BWDYT-11), two mutant lines (MASR-18 and MASR-64) developed through radiation-induced mutations, two doubled-haploid lines (DH-3/25 and DH8/13). The effects of water stress were determined on different phenological traits viz., days of booting, days of heading, grain filling period, days to maturity, and morphological yield associated traits viz., plant height, tillers per plant, spike length, grains per spike, main spike yield, seed index, and the grain yield per plot. Ten plants per replication/genotype and treatments were randomly selected to record the data. Result revealed that the genotypes, treatments, and genotype $\times$ treatment interactions were highly and significantly $(\mathrm{P} \leq 0.01)$ different with each other for most of the traits. However, the genotypes performed differently and variably with each other under water stress conditions imposed at various growth stages. Wheat genotypes BWDYT-2, BWDYT-3, BWDYT-4, and MASR-64 showed earlier maturity and could be more suitable under stress conditions. Genotypes BWDYT-1, BWDYT-5, BWDYT-6, BWDYT-8, BWDYT-10, BWDYT-11, MASR-64, DH3/25, and DH8/13required more days for grain filling period at $\mathrm{T} 1$; could be considered as tolerant.

Keywords: Drought tolerant; Phenological traits; Water stress; Wheat genotypes

\section{Introduction}

Water stress is a major factor affecting about $31.5 \%$ of 99 million hectares of developing countries, and at least 60 million hectares of wheat are cultivating in these area [1]. Therefore, improving the quality and quantity of crops is the central goal of agriculture research. Adding to its soil physical and chemical properties, and the absorption of nutrients can be enhanced by humic acids to improve root health that consequently increase plants yield [2]. Several areas around the world encounter water shortage during wheat growing season. Understandably, the world population is increasing very fast particularly in developing countries and ultimate increase of food supply is parallel such increase of food demands can be fulfilled from rain-fed areas [3]. 
[M1]The first citation should be number one and the next citation should be the next number Wheat (Triticum aestivum L.) is a staple food and the major cereal grain crop in the world, including Pakistan. Wheat is the second major crop cultivated throughout the temperate and tropical regions of the world. It provides more than $50 \%$ of the total calories and $60 \%$ of the total protein consumed by the population as a whole. Being the member of grass family, wheat dominates world agricultural production, because it directly or indirectly provides a large proportion for the human diet [4]. It contributes 10.6 percent to the value added in agriculture and $2.6 \%$ to grass domestic production.

Nevertheless, number of studies have been documented on wheat under water stress tolerance globally. Water stress tolerance level varies from species to species or even within species [5] stated that water deficit and salt stress are global problems that affect the survival of agricultural vegetation and sustainable meals manufacturing.

Water stress tolerance is to be considered as main breeding target in evaluating the crop performance under water deficit conditions [6]. The plant response to drought can be studied by identification of traits related to drought tolerance at the morphological, physiological, and biochemical levels [7, 8]. The water stress is the most important environmental cause major losses to the crops and can decrease grain yield, therefore approximately 17 to $70 \%$ grain yield losses have been reported [9]. Therefore, understanding selection of the biochemical and physiological origin of water stress tolerance in plants is essential for crops breeding [10].

Wheat is the most important cereal crop of the world and major staple food crop of Pakistan. Grain yield is the polygenic complex character governed by the genotype, environment and genotype $\mathrm{x}$ environmental $(\mathrm{GxE})$ interaction. Pakistan ranked in top10 wheat producing countries of the world [11]. However, the appropriate yields are not being obtained due to biotic (diseases and pests) and abiotic stresses which includes shortage of irrigation water (drought), heat stresses and the salinity [12]. The identification of new ideotypes possesses tolerance to water stress conditions is the one way to minimize the yield gap. These all environmental stresses, water stress is the main environmental constraints such as hot and dry weather limiting the crop productivity of wheat. Due to very rare rainfalls in the in generally country and particularly in Sindh, there is acute scarcity of the irrigation water in the canals especially during rabi crop season. The development of germplasm endowed with better tolerance to such biotic and abiotic stresses through various breeding techniques is one of the main objectives of the plant breeding. The identification of new ideotypes possesses tolerance to water stress conditions is the one way to minimize the yield gap. Keeping in view the shortage of irrigation water, the experiment was conducted to estimate the effects of water stresses on various yield and yield associated traits and to select the potential wheat genotypes for future breeding.

The current study was conducted to screening out the 17 genotype of wheat under water stress condition at different growth stages from seedling to maturity to see the effects of water stress on phenological and morphological traits among the varieties. The findings of wheat genotypes under water stress conditions enable to provide information regarding the diversity of new elite germplasm and their performance in severe environments effects of water stress on cultivar at different growth stages.

\section{Materials and Methods \\ Location and cropping history of experimental area}

The study was conducted to evaluate the effect of water stress on fifteen newly developed bread wheat (Triticum aestivum L.) genotypes comprised of 11 exotic genotypes (BWDYT-1 to BWDYT-11), two mutant lines (MASR-18 and MASR64) developed through radiation-induced 
mutations, two doubled-haploid lines (DH$3 / 25$ and DH8/13) and two standard varieties viz., NIA-Amber (droughtsensitive), Khirman (drought-tolerant). All genotypes were evaluated using various water stresses levels under field conditions. Experiment was conducted in three treatments with 3 replications in Split Plot Design (SPD) at Nuclear Institute of Agriculture (NIA) Tandojam, Sindh Pakistan, during wheat growing season 2017-18. The experiment was conducted using three different water stress conditions imposed at various critical growth stages viz., $\mathrm{T} 1=$ treatment 1 (single irrigation), T2 $=$ treatment 2 (two irrigations), and control $\mathrm{T} 3$ = treatment 3 (four irrigations), (Table 1). Each genotype was grown with four rows, $3 \mathrm{~m}$ long and $30 \mathrm{~cm}$ apart between rows. Plot size was $3.6 \mathrm{~m}^{2}(3 \mathrm{~m} \times 1.2 \mathrm{~m})$. Data was recorded on various phenological traits viz., days of booting, days of heading, grain filling period, days to maturity, and morphological yield associated traits viz., plant height $(\mathrm{cm})$, tillers plant $^{-1}$, spike length $(\mathrm{cm})$, grains spike ${ }^{-1}$, main spike yield $(\mathrm{g})$, seed index (1000-grain weight $\mathrm{g})$, and the grain yield $\operatorname{plot}^{-1}(\mathrm{~g})$.

Table 1. Schematic treatments for irrigation of wheat (Triticum aestivum L.) genotype under water stress conditions in three treatments

\begin{tabular}{|c|c|c|}
\hline No. of irrigation & Treatment 1 & Growth stage \\
\hline No. of irrigation applied & Time of application & Growth stage of crop \\
\hline Single irrigation & 14 days of sowing & Seedling \\
\hline \multicolumn{3}{|c|}{ Treatment 2 } \\
\hline No. of irrigation applied & Time of application & Growth stage of crop \\
\hline $\mathbf{1}^{\text {st }}$ irrigation & 14 days of sowing & seedling stage \\
\hline $\mathbf{2}^{\text {nd }}$ irrigation & 28 days of sowing & anthesis stage \\
\hline No. of irrigation applied & Treatment 3 \\
\hline $\mathbf{1}^{\text {st }}$ irrigation & Time of application & Growth stage of crop \\
\hline $\mathbf{2}^{\text {nd }}$ irrigation & 14 days of sowing & Seedling stage \\
\hline $\mathbf{3}^{\text {rd }}$ irrigation & 28 days of sowing & Booting stage \\
\hline $\mathbf{4}^{\text {th }}$ irrigation & 42 days of sowing & Milky stage \\
\hline
\end{tabular}

\section{Statistical analysis}

Analysis of variance (ANOVA) was applied to test split plot arrangement for wheat experiments with Statistix 8.1 software. Each treatment was compared at $\mathrm{P}<0.05$ level of probability using LSD test. Results

Significant effect on various yield and its components, genotypes, treatment and genotype $\times$ treatment interaction was highly and positively significant $(p<0.01)$ observed in this study. A significant decrease in various morphological traits was recorded in severe water stress condition (single irrigation treatment) as compare to rest of the treatments. A summary of overall comparisons between various pehnological traits as affected by different treatments are presented (Table 2).

\section{Phenological traits}

\section{Days to $75 \%$ booting}

The overall mean for days booting from all wheat genotypes among three treatments showed significantly differences $(\mathrm{P}<0.05)$. Treatment 3 (71.56) showed significantly increase in days of booting as compared to treatment 1 and treatment 2 (69.47 and 71.09) (Table 3; Fig. 1). Highly significant differences $(\mathrm{P}<0.05)$ for the trait were recorded in wheat genotypes under treatmentlduring the days of booting ranged from 64.0 in genotype BWDYT-8 and DH8/13 to 76.6 in genotype MASR-18. Eight test entries (BWDYT-1, BWDYT-3, BWDYT-4, BWDYT-5, BWDYT-6, BWDYT-7, BWDYT-9, MASR-64) and a controlled/check NIA-Amber variety showed significant $(\mathrm{P}<0.05)$ increase in days to booting at T1 (treatment1). Highly 
significant differences $(\mathrm{P}<0.05)$ for the trait days to booting were recorded in wheat genotypes at $\mathrm{T} 2$. At $\mathrm{T} 2$, days to booting ranged from 64.0 in genotype BWDYT-6 to 79.6 in genotype MASR-18. Four test entries (BWDYT-2, BWDYT-3, BWDYT7 and BWDYT-9) and a check variety NIAAmber showed significant $(\mathrm{P}<0.05)$ increase in days to booting at T2.Highly significant differences $(\mathrm{P}<0.05)$ for the trait days to booting were recorded in wheat genotypes at T3. At T3, days to booting ranged from 64.0 in genotype BWDYT- 8 to 77.0 in genotype NIA-Amber. Eleven test entries (BWDYT-1, BWDYT-2, BWDYT3, BWDYT-4, BWDYT-5, BWDYT-6, BWDYT-7, BWDYT-9, BWDYT-10, BWDYT-11, DH3/25) showed significantly $(\mathrm{P}<0.05)$ increase in days to booting at $\mathrm{T} 3$.

\section{Days to $75 \%$ heading}

The number of days to heading of all wheat genotypes among three water stress treatments showed significant differences $(\mathrm{P}<0.05)$. T2 and T3 (81.68 and 80.86) showed significant increase in days to heading as compared to T1 (78.94), (Table 4; Fig. 2).Highly significant differences
$(\mathrm{P}<0.05)$ for the trait days to heading were recorded in wheat genotypes at $\mathrm{T} 1$ (Table 2). At T1, days to heading ranged from 72.3 in genotype BWDYT-8 to 87.0 in genotype MASR-18. Six test entries (BWDYT-2, BWDYT-3, BWDYT-4, BWDYT-6, BWDYT-7, BWDYT-9) and a check variety NIA-Amber showed significant $(\mathrm{P}<0.05)$ increase in days to heading at $\mathrm{T} 1$. The trait days to heading at T2 showed significant difference in wheat genotypes at $\mathrm{T} 2$. At $\mathrm{T} 2$, days to heading ranged from 72.3 in genotype BWDYT-8 to 87.0 in genotype MASR-18. Five test entries (BWDYT-2, BWDYT-3, BWDYT-4, BWDYT-7, BWDYT-9) and a check variety NIA-Amber showed significantly $(\mathrm{P}<0.05)$ increase in days to heading at T2.Highly significant differences $(\mathrm{P}<0.05)$ in days to heading were recorded in wheat genotypes at T3. At T3, days to heading ranged from 72.3 in genotype BWDYT-8 to 87.0 in genotype MASR-18. Eight test entries (BWDYT-1, BWDYT-2, BWDYT4, BWDYT-5, BWDYT-6, BWDYT-7, BWDYT-9, BWDYT-11) and a check variety NIA-Amber showed significantly $(\mathrm{P}<0.05)$ increase in days to heading at $\mathrm{T} 3$.

Table 2. Overall mean squares (MS) from pooled ANOVA of different phenological traits of wheat genotypes as affected under different water stress conditions

\begin{tabular}{|c|c|c|c|c|c|}
\hline \multirow{2}{*}{ Source of variation } & \multirow{2}{*}{ D.F. } & \multicolumn{5}{|c|}{ Mean Squares (M.S) } \\
\cline { 3 - 6 } & 2 & 100.5 & 134.9 & 1.418 & 149.0 \\
\hline Replications & 16 & $114.5^{* *}$ & $174.2^{* *}$ & 4.958 & $167.6^{* *}$ \\
\hline Genotypes & 32 & 33.5 & 39.9 & 4.231 & 39.3 \\
\hline Error (Rep x Genotype) & 2 & $61.8^{*}$ & $101.2^{* *}$ & $180.8^{* *}$ & 27.5 \\
\hline Treatments & 32 & 17.0 & 11.3 & 3.831 & 10.6 \\
\hline Genotype x Treatment interaction & 68 & 19.212 & 11.02 & 3.31 & 12.542 \\
\hline Error & 152 & -- & -- & -- & -- \\
\hline Total & & & - & D. H & G. F. P \\
\hline
\end{tabular}

*Significant at 0.05 level of probability, **Significant at 0.01 level of probability. $\mathrm{D} . \mathrm{B}=$ days to booting; $\mathrm{D} . \mathrm{H}=$ days to heading; D.M= days to maturity; G.F.P= grain filling period 
Table 3. Overall mean performance for Days to $75 \%$ booting of wheat genotypes as affected by three different water stress conditions

\begin{tabular}{|c|c|c|c|c|c|}
\hline \multirow{2}{*}{ Genotypes } & \multicolumn{5}{|c|}{ Days to 75\% Boting } \\
\cline { 2 - 6 } & $\mathbf{T 1}$ & $\mathbf{T 2}$ & $\mathbf{T 3}$ & $\begin{array}{c}\text { T1\% } \\
\text { increase/ } \\
\text { decrease over } \\
\text { control T3 }\end{array}$ & $\begin{array}{c}\text { T2\% increase/ } \\
\text { decrease over } \\
\text { control T3 }\end{array}$ \\
\hline BWDYT-1 & $71.3 \mathrm{ABCD}$ & $71.6 \mathrm{BCDE}$ & $72.6 \mathrm{AB}$ & -1.79 & -1.38 \\
\hline BWDYT-2 & $64.0 \mathrm{D}$ & $76.3 \mathrm{AB}$ & $74.0 \mathrm{AB}$ & -13.51 & 3.11 \\
\hline BWDYT-3 & $74.0 \mathrm{AB}$ & $74.6 \mathrm{ABC}$ & $70.6 \mathrm{ABC}$ & 4.82 & 5.67 \\
\hline BWDYT-4 & $73.6 \mathrm{ABC}$ & $71.0 \mathrm{BCDE}$ & $75.0 \mathrm{AB}$ & -1.87 & -5.33 \\
\hline BWDYT-5 & $72.6 \mathrm{ABCD}$ & $69.3 \mathrm{BCDE}$ & $72.6 \mathrm{AB}$ & 0.00 & -4.55 \\
\hline BWDYT-6 & $70.6 \mathrm{ABCD}$ & $64.0 \mathrm{E}$ & $70.3 \mathrm{ABC}$ & 0.43 & -8.96 \\
\hline BWDYT-7 & $74.0 \mathrm{AB}$ & $73.6 \mathrm{ABCD}$ & $72.6 \mathrm{AB}$ & 1.93 & 1.38 \\
\hline BWDYT-8 & $64.0 \mathrm{D}$ & $66.0 \mathrm{DE}$ & $64.0 \mathrm{C}$ & 0.00 & 3.13 \\
\hline BWDYT-9 & $72.3 \mathrm{ABCD}$ & $76.3 \mathrm{AB}$ & $75.0 \mathrm{AB}$ & -3.60 & 1.73 \\
\hline BWDYT-10 & $66.0 \mathrm{BCD}$ & $70.0 \mathrm{BCDE}$ & $70.6 \mathrm{ABC}$ & -6.52 & -0.85 \\
\hline BWDYT-11 & $66.0 \mathrm{BCD}$ & $70.3 \mathrm{BCDE}$ & $70.3 \mathrm{ABC}$ & -6.12 & 0.00 \\
\hline MASR-18 & $76.6 \mathrm{~A}$ & $79.6 \mathrm{~A}$ & $76.6 \mathrm{~A}$ & 0.00 & 3.92 \\
\hline MASR-64 & $68.0 \mathrm{ABCD}$ & $68.0 \mathrm{CDE}$ & $68.0 \mathrm{BC}$ & 0.00 & 0.00 \\
\hline DH3/25 & $65.0 \mathrm{CD}$ & $66.0 \mathrm{DE}$ & $70.0 \mathrm{ABC}$ & -7.14 & -5.71 \\
\hline DH8/13 & $64.0 \mathrm{D}$ & $68.0 \mathrm{CDE}$ & $68.0 \mathrm{BC}$ & -5.88 & 0.00 \\
\hline Khirman & $66.0 \mathrm{BCD}$ & $67.3 \mathrm{CDE}$ & $69.0 \mathrm{BC}$ & -4.35 & -2.46 \\
\hline NIA-Amber & $72.6 \mathrm{ABCD}$ & $76.3 \mathrm{AB}$ & $77.0 \mathrm{~A}$ & -5.71 & -0.91 \\
\hline Overall mean & $69.47 \mathrm{~B}$ & $71.09 \mathrm{AB}$ & $71.56 \mathrm{~A}$ & -2.92 & -0.66 \\
\hline $\begin{array}{c}\text { \% increase/ } \\
\text { decrease over } \\
\text { control }\end{array}$ & -2.92 & -0.65 & - & & \\
\hline
\end{tabular}

*T1=Single irrigation. T2=Two irrigation, T3=Four irrigation

Phenological trait of wheat genotype (Triticum aestivum L.) under three water stress treatments

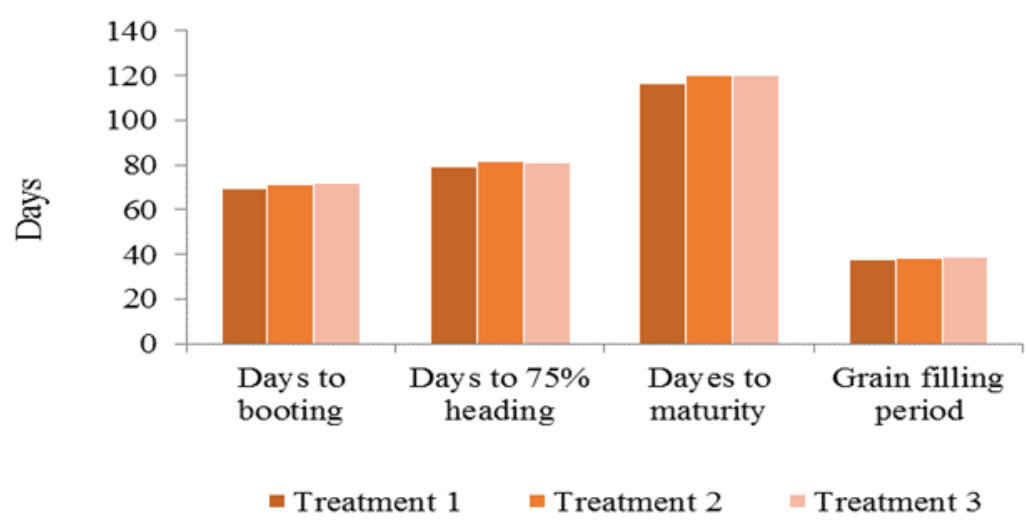

Figure 1. Comparative mean performance of phenological traits of wheat (Triticum aestivum $\mathbf{L}$.) genotypes under three water stress treatments 
Table 4. Overall mean performance for days to $75 \%$ heading of wheat genotypes as affected by three different water stress conditions

\begin{tabular}{|c|c|c|c|c|c|}
\hline \multirow[b]{2}{*}{ Genotypes } & \multicolumn{5}{|c|}{ Days to $75 \%$ Heading } \\
\hline & T1 & $\mathbf{T 2}$ & T3 & $\begin{array}{c}\text { T1\% } \\
\text { increase/ } \\
\text { decrease } \\
\text { over control } \\
\mathrm{T} 3 \\
\end{array}$ & $\begin{array}{c}\text { T2\% } \\
\text { increase/ } \\
\text { decrease } \\
\text { over control } \\
\text { T3 }\end{array}$ \\
\hline BWDYT-1 & $79.0 \mathrm{BCDEF}$ & $81.0 \mathrm{BCDEF}$ & $81.0 \mathrm{ABCD}$ & -2.47 & 0.00 \\
\hline BWDYT-2 & $85.0 \mathrm{AB}$ & $87.6 \mathrm{AB}$ & 82.0 ABCD & 3.66 & 6.83 \\
\hline BWDYT-3 & 81.6 ABCD & $84.3 \mathrm{ABCDE}$ & $80.3 \mathrm{BCDE}$ & 1.62 & 4.98 \\
\hline BWDYT-4 & $84.0 \mathrm{AB}$ & 85.667 ABC & $84.3 \mathrm{ABC}$ & -0.36 & 1.62 \\
\hline BWDYT-5 & $79.3 \mathrm{BCDEF}$ & $77.3 \mathrm{DEF}$ & 81.3 ABCD & -2.46 & -4.92 \\
\hline BWDYT-6 & $80.0 \mathrm{ABCDE}$ & $76.6 \mathrm{EF}$ & $82.0 \mathrm{ABCD}$ & -2.44 & -6.59 \\
\hline BWDYT-7 & $83.3 \mathrm{ABC}$ & $89.0 \mathrm{AB}$ & $85.6 \mathrm{AB}$ & -2.69 & 3.97 \\
\hline BWDYT-8 & $72.3 \mathrm{~F}$ & $73.3 \mathrm{~F}$ & $73.6 \mathrm{E}$ & -1.77 & -0.41 \\
\hline BWDYT-9 & $83.3 \mathrm{ABC}$ & $89.6 \mathrm{~A}$ & $85.0 \mathrm{AB}$ & -2.00 & 5.41 \\
\hline BWDYT-10 & 75.0 DEF & 81.0 BCDEF & 78.6 BCDE & -4.58 & 3.05 \\
\hline BWDYT-11 & $73.6 \mathrm{EF}$ & 79.0 CDEF & 83.0 ABCD & -11.33 & -4.82 \\
\hline MASR-18 & $87.0 \mathrm{~A}$ & $89.6 \mathrm{~A}$ & $88.0 \mathrm{~A}$ & -1.14 & 1.82 \\
\hline MASR-64 & 76.3 CDEF & 79.0 CDEF & $77.0 \mathrm{DE}$ & -0.91 & 2.60 \\
\hline DH3/25 & $75.0 \mathrm{DEF}$ & 77.6 CDEF & $77.3 \mathrm{CDE}$ & -2.98 & 0.39 \\
\hline DH8/13 & $72.6 \mathrm{~F}$ & $75.0 \mathrm{~F}$ & $76.3 \mathrm{DE}$ & -4.85 & -1.70 \\
\hline Khirman & $73.6 \mathrm{EF}$ & 77.6 CDEF & $76.3 \mathrm{DE}$ & -3.54 & 1.70 \\
\hline NIA-Amber & 80.6 ABCDE & 85.0 ABCD & 82.6 ABCD & -2.42 & 2.91 \\
\hline Overall mean & $78.94 \mathrm{~B}$ & $81.68 \mathrm{~A}$ & $80.86 \mathrm{~A}$ & -2.37 & 1.01 \\
\hline $\begin{array}{c}\% \text { increase/ } \\
\text { decrease over } \\
\text { control }\end{array}$ & -2.37 & 1.01 & -- & & \\
\hline
\end{tabular}

*Treatment $1=$ Single irrigation. Treatment $2=$ Two irrigation, Treatment $3=$ Four irrigation

Morphological traits at three water stress treatments of wheat (Triticum aestivum L.
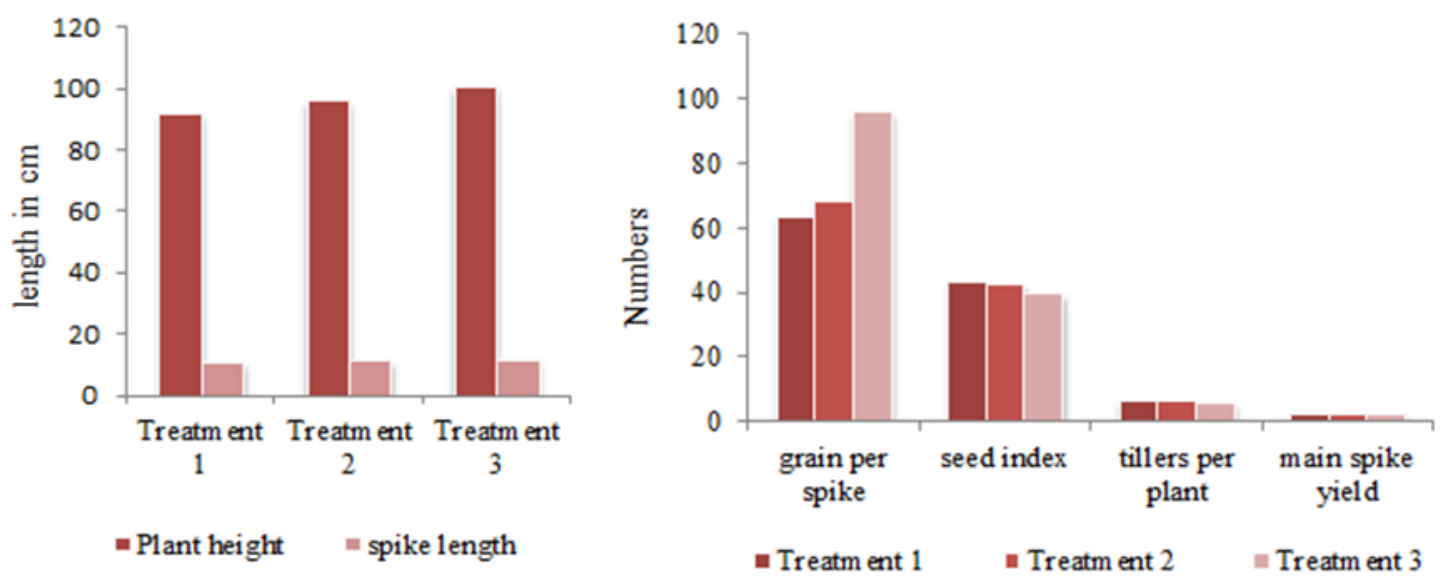

Figure 2. Comparative mean performance of morphological traits of wheat (Triticum aestivum $\mathrm{L}$.) under three water stress treatments 


\section{Days to $75 \%$ maturity}

Days to maturity of all wheat genotypes among three treatments showed significant difference $(\mathrm{P}<0.05)$. T2 and T3 (119.78 and 120.02) showed significant increase in days to maturity as compared to $\mathrm{T} 1$ (116.65) (Table 5; Fig. 3). Non-significant differences for the trait days to maturity were recorded in wheat genotypes at T1. At T1 days to maturity ranged from 115.0 to 118.6 in genotype BWDYT-8 and in BWDYT-9. Highly significant differences $(\mathrm{P}<0.05)$ for the trait days to maturity were recorded in wheat genotypes at T2. At T2, days to maturity ranged from 118.0 in genotype BWDYT-3 to 122.0 in genotype MASR-18. Four test entries (BWDYT-2, BWDYT-3, BWDYT-4 and MASR-64) matured earlier (118 days) than all other contesting genotypes. Highly significant differences $(\mathrm{P}<0.05)$ for the trait days to maturity were recorded in wheat genotypes at T3. At T3, days to maturity ranged from 117.3 I genotype BWDYT-2 to 122.0 in genotype MASR-64. Three test entries (BWDYT-2, BWDYT-7 and Dh-3/25) attain maturity earlier (118 days) than all other entries.

Table 5. Overall mean performance for Days to $75 \%$ maturity of wheat genotypes as affected by three different water stress conditions

\begin{tabular}{|c|c|c|c|c|c|}
\hline & \multicolumn{5}{|c|}{ Days to 75\% maturity } \\
\cline { 2 - 6 } Genotypes & $\mathbf{T 1}$ & $\mathbf{T 2}$ & $\mathbf{T 3}$ & $\begin{array}{c}\text { T1\% increase/ } \\
\text { decrease over } \\
\text { control T3 }\end{array}$ & $\begin{array}{c}\text { T2\% increase/ } \\
\text { decrease over } \\
\text { control T3 }\end{array}$ \\
\hline BWDYT-1 & $116.3 \mathrm{~A}$ & $119.0 \mathrm{BCD}$ & $119.3 \mathrm{ABCD}$ & -2.51 & -0.25 \\
\hline BWDYT-2 & $117.0 \mathrm{~A}$ & $118.3 \mathrm{D}$ & $117.3 \mathrm{D}$ & -0.26 & 0.85 \\
\hline BWDYT-3 & $115.6 \mathrm{~A}$ & $118.0 \mathrm{D}$ & $119.3 \mathrm{ABCD}$ & -3.10 & -1.09 \\
\hline BWDYT-4 & $116.6 \mathrm{~A}$ & $118.6 \mathrm{CD}$ & $120.0 \mathrm{ABCD}$ & -2.83 & -1.17 \\
\hline BWDYT-5 & $118.3 \mathrm{~A}$ & $119.6 \mathrm{ABCD}$ & $120.0 \mathrm{ABCD}$ & -1.42 & -0.33 \\
\hline BWDYT-6 & $116.3 \mathrm{~A}$ & $119.3 \mathrm{ABCD}$ & $121.6 \mathrm{~A}$ & -4.36 & -1.89 \\
\hline BWDYT-7 & $116.0 \mathrm{~A}$ & $121.3 \mathrm{ABC}$ & $118.3 \mathrm{CD}$ & -1.94 & 2.54 \\
\hline BWDYT-8 & $115.0 \mathrm{~A}$ & $119.6 \mathrm{ABCD}$ & $120.3 \mathrm{ABC}$ & -4.41 & -0.58 \\
\hline BWDYT-9 & $118.6 \mathrm{~A}$ & $120.3 \mathrm{ABCD}$ & $120.0 \mathrm{ABCD}$ & -1.17 & 0.25 \\
\hline BWDYT-10 & $116.6 \mathrm{~A}$ & $119.3 \mathrm{ABCD}$ & $120.6 \mathrm{ABC}$ & -3.32 & -1.08 \\
\hline BWDYT-11 & $115.6 \mathrm{~A}$ & $119.6 \mathrm{ABCD}$ & $121.3 \mathrm{AB}$ & -4.70 & -1.40 \\
\hline MASR-18 & $118.3 \mathrm{~A}$ & $122.0 \mathrm{~A}$ & $121.6 \mathrm{~A}$ & -2.71 & 0.33 \\
\hline MASR-64 & $115.6 \mathrm{~A}$ & $118.6 \mathrm{CD}$ & $122.0 \mathrm{~A}$ & -5.25 & -2.79 \\
\hline DH3/25 & $117.3 \mathrm{~A}$ & $119.0 \mathrm{BCD}$ & $118.6 \mathrm{BCD}$ & -1.10 & 0.34 \\
\hline DH8/13 & $115.3 \mathrm{~A}$ & $121.6 \mathrm{AB}$ & $120.0 \mathrm{ABCD}$ & -3.92 & 1.33 \\
\hline Khirman & $117.6 \mathrm{~A}$ & $120.3 \mathrm{ABCD}$ & $119.6 \mathrm{ABCD}$ & -1.67 & 0.59 \\
\hline NIA-Amber & $116.3 \mathrm{~A}$ & $121.3 \mathrm{ABC}$ & $120.0 \mathrm{ABCD}$ & -3.08 & 1.08 \\
\hline Overall mean & $116.65 \mathrm{~B}$ & $119.78 \mathrm{~A}$ & $120.02 \mathrm{~A}$ & -2.81 & -0.20 \\
\hline $\begin{array}{c}\text { \% increase/ } \\
\text { decrease over } \\
\text { control }\end{array}$ & -2.80 & -0.19 & & & \\
\hline
\end{tabular}

*Treatment $1=$ Single irrigation. Treatment $2=$ Two irrigation, Treatment $3=$ Four irrigation 


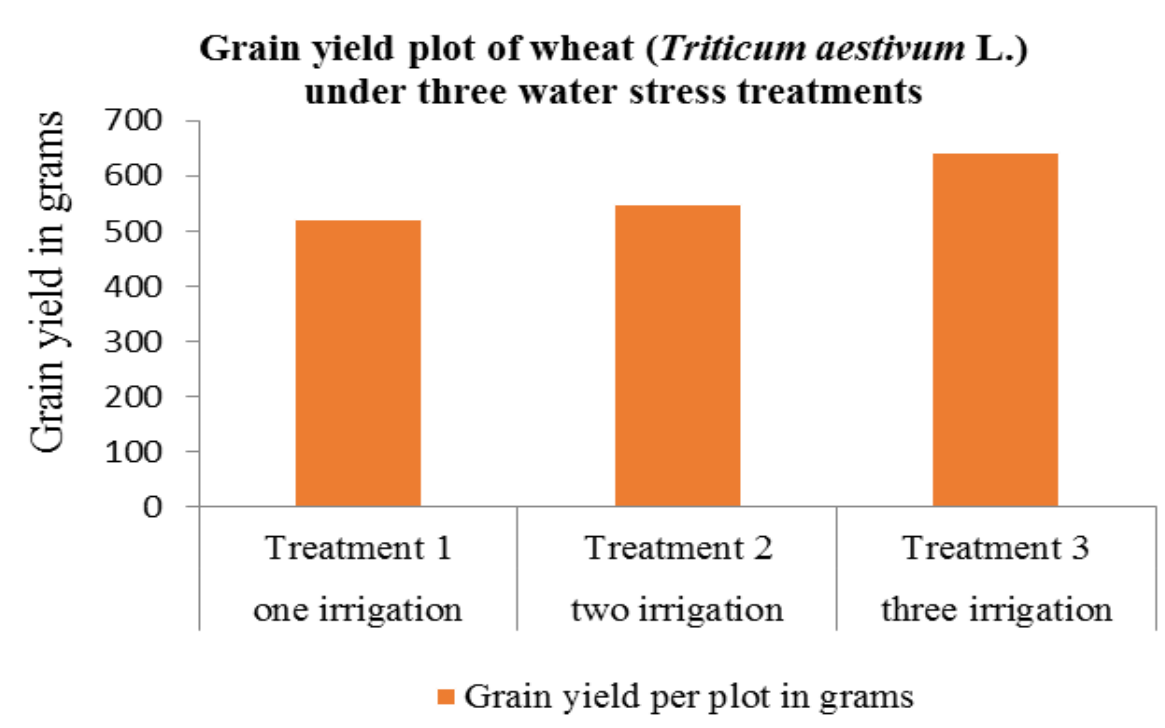

Figure 3. Comparative mean performance of grain yield per plot of wheat (Triticum aestivum $\mathrm{L}$.) under three water stress treatments

\section{Days to grain filling period}

The grain filling period of all wheat genotypes among three treatments showed significant difference $(\mathrm{P}<0.05)$. T3 (39.15) $\mathrm{T} 1$ and $\mathrm{T} 2$ showed significant increase in days to grain filling period (37.74 and 38.09), respectively (Table 6). At $\mathrm{T} 1$ highly significant differences $(\mathrm{P}<0.05)$ of the trait days to grain filling period were recorded in all wheat genotypes. At T1, days to grain filling period ranged from 31.3 in genotype MASR-18 to 44.0 in genotype Khirman. Nine test entries (BWDYT-1, BWDYT-5, BWDYT-6, BWDYT-8, BWDYT-10, BWDYT-11, MASR-64, DH3/25, DH8/13) took significantly $(\mathrm{P}<0.05)$ more days to grain filling period at T1. Highly significant differences $(\mathrm{P}<0.05)$ for the trait days to grain filling period were recorded in all wheat genotypes at T2. At T2, days to grain filling period ranged from 30.6 in genotype BWDYT-2 and BWDYT-9 to 46.6 in genotype DH8/13. Eight test entries
(BWDYT-1， BWDYT-5, BWDYT-6, BWDYT-8, BWDYT-10, BWDYT-11, MASR-64, DH3/25) and a check variety Khirman took $(\mathrm{P}<0.05)$ more days to grain filling period at T2. Highly significant differences $(\mathrm{P}<0.05)$ for the trait days to grain filling period were recorded in wheat genotypes at T3. At T3, days to grain filling period ranged from 32.6 I genotype BWDYT-7 to 46.6 in genotype BWDYT-8. Five test entries (BWDYT-6, BWDYT-10, MASR-64, DH3/25, DH8/13) and a check variety Khirman showed significantly $(\mathrm{P}<0.05)$ increase for days to grain filling period at $\mathrm{T} 3$.

\section{Morphological traits}

A significant decrease in various morphological traits was recorded in severe water stress condition (single irrigation treatment) as compare to rest of the treatments. A summary of overall comparisons between various morphological traits as affected by different treatments are presented (Table 7). 
Table 6. Overall mean performance for days to grain filling period of wheat genotypes as affected by three different water stress conditions

\begin{tabular}{|c|c|c|c|c|c|}
\hline \multirow[b]{2}{*}{ Genotypes } & \multicolumn{5}{|c|}{ Days to grain filling period } \\
\hline & T1 & $\mathbf{T 2}$ & T3 & $\begin{array}{c}\text { T1\% } \\
\text { increase/ } \\
\text { decrease } \\
\text { over control } \\
\text { T3 }\end{array}$ & $\begin{array}{c}\text { T2\% } \\
\text { increase/ } \\
\text { decrease } \\
\text { over control } \\
\text { T3 }\end{array}$ \\
\hline BWDYT-1 & 37.3 ABCDEF & 38.0 ABCDEF & $38.3 \mathrm{BCDE}$ & -2.61 & -0.78 \\
\hline BWDYT-2 & $32.0 \mathrm{EF}$ & $30.6 \mathrm{~F}$ & $35.3 \mathrm{DE}$ & -9.35 & -13.31 \\
\hline BWDYT-3 & $33.6 \mathrm{DEF}$ & $33.6 \mathrm{CDEF}$ & $39.0 \mathrm{BCDE}$ & -13.85 & -13.85 \\
\hline BWDYT-4 & 32.6 DEF & 33.0 DEF & $35.6 \mathrm{DE}$ & -8.43 & -7.30 \\
\hline BWDYT-5 & 39.0 ABCDE & $42.3 \mathrm{ABC}$ & 38.6 BCDE & 1.04 & 9.59 \\
\hline BWDYT-6 & $37.3 \mathrm{ABCDEF}$ & $42.6 \mathrm{AB}$ & $\begin{array}{c}39.6 \\
\text { ABCDE }\end{array}$ & -5.81 & 7.58 \\
\hline BWDYT-7 & $32.6 \mathrm{DEF}$ & $32.3 \mathrm{EF}$ & $32.6 \mathrm{E}$ & 0.00 & -0.92 \\
\hline BWDYT-8 & $42.6 \mathrm{AB}$ & $46.3 \mathrm{~A}$ & $46.6 \mathrm{~A}$ & -8.58 & -0.64 \\
\hline BWDYT-9 & $35.3 \mathrm{CDEF}$ & $30.6 \mathrm{~F}$ & $35.0 \mathrm{DE}$ & 0.86 & -12.57 \\
\hline BWDYT-10 & $41.6 \mathrm{ABC}$ & $38.3 \mathrm{ABCDEF}$ & 42.0 ABCD & -0.95 & -8.81 \\
\hline BWDYT-11 & $42.0 \mathrm{ABC}$ & 40.6 ABCDE & $38.3 \mathrm{BCDE}$ & 9.66 & 6.01 \\
\hline MASR-18 & $31.3 \mathrm{~F}$ & $32.3 \mathrm{EF}$ & $33.6 \mathrm{E}$ & -6.85 & -3.87 \\
\hline MASR-64 & 39.3 ABCD & 39.6 ABCDE & $45.0 \mathrm{AB}$ & -12.67 & -12.00 \\
\hline DH3/25 & $42.3 \mathrm{ABC}$ & 41.3 ABCD & 41.3 ABCD & 2.42 & 0.00 \\
\hline DH8/13 & $42.6 \mathrm{AB}$ & $46.6 \mathrm{~A}$ & $43.6 \mathrm{ABC}$ & -2.29 & 6.88 \\
\hline Khirman & $44.0 \mathrm{~A}$ & $42.6 \mathrm{AB}$ & $43.3 \mathrm{ABC}$ & 1.62 & -1.62 \\
\hline NIA-Amber & $35.6 \mathrm{BCDEF}$ & $36.3 \mathrm{BCDEF}$ & $37.3 \mathrm{CDE}$ & -4.56 & -2.68 \\
\hline Overall mean & $37.74 \mathrm{~B}$ & $38.09 \mathrm{AB}$ & $39.15 \mathrm{~A}$ & -3.60 & -2.71 \\
\hline $\begin{array}{c}\text { \% increase/ } \\
\text { decrease over } \\
\text { control }\end{array}$ & -3.60 & -2.70 & -- & & \\
\hline
\end{tabular}

*Treatment $1=$ Single irrigation. Treatment2=Two irrigation, Treatment $3=$ Four irrigation

Table 7. Overall mean squares (MS) from pooled ANOVA of different morphological traits of wheat genotypes as affected by different water stress conditions.

\begin{tabular}{|c|c|c|c|c|c|c|c|c|}
\hline \multirow{2}{*}{ Source of variation } & \multirow{2}{*}{ D.F. } & P.H & T.P & S.L & Gr./S & $\begin{array}{c}\text { M.S. } \\
\text { Y }\end{array}$ & T.G & G.Y \\
\hline Replications & 2 & 3.64 & 1.64 & 0.83 & 3.23 & 0.03 & 149.8 & 72.1 \\
\hline Genotypes & 16 & $\begin{array}{c}211.5^{*} \\
*\end{array}$ & $\begin{array}{c}3.64^{*} \\
*\end{array}$ & $\begin{array}{c}3.3^{*} \\
*\end{array}$ & 143.2 & $0.4 * *$ & 59.5 & 108.1 \\
\hline Error (Rep x Genotype) & 32 & 9.77 & 2.47 & 0.42 & 32.09 & 0.07 & 64.1 & 76.8 \\
\hline Treatments & 2 & 947.5 & 6.61 & 9.06 & $\begin{array}{c}502.0 \\
7\end{array}$ & 0.50 & $\begin{array}{c}200.8 \\
*\end{array}$ & 109.2 \\
\hline $\begin{array}{c}\text { Genotype x Treatment } \\
\text { interaction }\end{array}$ & 32 & 13.5 & 1.17 & 0.43 & 64.92 & 0.05 & $\begin{array}{c}5296 \\
1\end{array}$ & $\begin{array}{c}115.0^{*} \\
*\end{array}$ \\
\hline Error & 68 & 8.6 & 1.30 & 0.37 & 57.04 & 0.07 & $\begin{array}{c}3946 \\
5\end{array}$ & 54.15 \\
\hline Total & 152 & -- & -- & -- & -- & -- & -- & -- \\
\hline *Significant at 0.05 level of probability, **Significant at 0.01 level of probability. \\
\hline
\end{tabular}

$\mathrm{PH}=$ plant height; $\mathrm{T} . \mathrm{P}=$ tillers plant $^{-1} ; \mathrm{S} . \mathrm{L}=$ spike length; $\mathrm{Gr} . / \mathrm{S}=$ grains spike $^{-1} \mathrm{MSY}=$ main spike yield; $\mathrm{T} . \mathrm{G}=$ thousand grains weight; G.Y= grain yield plot $^{-1}$ 


\section{Plant height (cm)}

Height of plant showed significant difference for wheat genotypes among three treatments $(\mathrm{P}<0.05)$. T3 $\quad(100.44 \mathrm{~cm})$. Significant increase in plant height was compared to T1 and T2 (91.82 and $96.0 \mathrm{~cm})$ respectively. Highly significant differences $(\mathrm{P}<0.05)$ for the trait of plant height were recorded in wheat genotypes at T1 (Table 8). At T1, plant height ranged from $82.07 \mathrm{~cm}$ in genotype BWDYT-9 to $103.33 \mathrm{~cm}$ in genotype BWDYT-2. Six test entries (BWDYT-1, BWDYT-5, BWDYT8, BWDYT-11, MASR-64, DH8/13) showed significantly $(\mathrm{P}<0.05)$ increase in plant height at $\mathrm{T} 1$. At $\mathrm{T} 2$, plant height ranged from $89.18 \mathrm{~cm}$ to $106.00 \mathrm{~cm}$ in genotype BWDYT-6 and genotype BWDYT-2. Two test entries (BWDYT-11 and MASR-64) showed significantly $(\mathrm{P}<0.05)$ increase in plant height at $\mathrm{T} 2$. At T3, plant height ranged from $92.23 \mathrm{~cm}$ to $112.60 \mathrm{~cm}$ in BWDYT-10 and BWDYT-2. Five test entries BWDYT-2, BWDYT-8, BWDYT-11, MASR-64, DH8/13 and a check variety Khirman showed significantly $(\mathrm{P}<0.05)$ increase for plant height at $\mathrm{T} 3$.

Table 8. Overall mean performance for Plant height $(\mathrm{cm})$ of wheat genotypes as affected by 3 different water stress conditions

\begin{tabular}{|c|c|c|c|c|c|}
\hline \multirow{2}{*}{ Genotypes } & \multicolumn{5}{|c|}{ Plant height (cm) } \\
\cline { 2 - 6 } & $\mathbf{T 1}$ & $\mathbf{T 2}$ & $\mathbf{T 3}$ & $\begin{array}{c}\text { T1\% } \\
\text { increase/ } \\
\text { decrease } \\
\text { over } \\
\text { control T3 }\end{array}$ & $\begin{array}{c}\text { T2\% } \\
\text { increase/ } \\
\text { decrease } \\
\text { over } \\
\text { control T3 }\end{array}$ \\
\hline BWDYT-1 & $95.93 \mathrm{BC}$ & $98.60 \mathrm{CD}$ & $100.27 \mathrm{CD}$ & -4.33 & -1.67 \\
\hline BWDYT-2 & $103.33 \mathrm{~A}$ & $106.00 \mathrm{~A}$ & $112.60 \mathrm{~A}$ & -8.23 & -5.86 \\
\hline BWDYT-3 & $84.03 \mathrm{HI}$ & $89.68 \mathrm{HI}$ & $95.07 \mathrm{FG}$ & -11.61 & -5.67 \\
\hline BWDYT-4 & $90.70 \mathrm{EF}$ & $92.71 \mathrm{FGH}$ & $94.43 \mathrm{FG}$ & -3.95 & -1.82 \\
\hline BWDYT-5 & $96.77 \mathrm{~B}$ & $98.57 \mathrm{CD}$ & $100.77 \mathrm{CD}$ & -3.97 & -2.18 \\
\hline BWDYT-6 & $86.73 \mathrm{FGH}$ & $89.18 \mathrm{I}$ & $96.23 \mathrm{EF}$ & -9.87 & -7.33 \\
\hline BWDYT-7 & $88.37 \mathrm{FG}$ & $97.35 \mathrm{D}$ & $102.33 \mathrm{BC}$ & -13.64 & -4.87 \\
\hline BWDYT-8 & $94.50 \mathrm{BCD}$ & $96.17 \mathrm{DE}$ & $105.48 \mathrm{~B}$ & -10.41 & -8.83 \\
\hline BWDYT-9 & $82.07 \mathrm{I}$ & $90.90 \mathrm{GHI}$ & $97.63 \mathrm{DEF}$ & -15.94 & -6.89 \\
\hline BWDYT-10 & $86.67 \mathrm{GH}$ & $89.87 \mathrm{HI}$ & $92.23 \mathrm{G}$ & -6.03 & -2.56 \\
\hline BWDYT-11 & $96.23 \mathrm{BC}$ & $102.30 \mathrm{~B}$ & $104.15 \mathrm{~B}$ & -7.60 & -1.78 \\
\hline MASR-18 & $92.63 \mathrm{CDE}$ & $93.58 \mathrm{EFG}$ & $98.47 \mathrm{DE}$ & -5.93 & -4.97 \\
\hline MASR-64 & $95.57 \mathrm{BC}$ & $101.57 \mathrm{BC}$ & $104.07 \mathrm{~B}$ & -8.17 & -2.40 \\
\hline DH3/25 & $92.80 \mathrm{CDE}$ & $96.09 \mathrm{DE}$ & $99.77 \mathrm{CD}$ & -6.99 & -3.69 \\
\hline DH8/13 & $97.04 \mathrm{~B}$ & $97.31 \mathrm{D}$ & $104.07 \mathrm{~B}$ & -6.76 & -6.50 \\
\hline Khirman & $90.18 \mathrm{EFG}$ & $98.30 \mathrm{D}$ & $104.60 \mathrm{~B}$ & -13.79 & -6.02 \\
\hline NIA-Amber & $87.43 \mathrm{FGH}$ & $94.03 \mathrm{EF}$ & $95.37 \mathrm{EFG}$ & -8.33 & -1.41 \\
\hline Overall mean & $91.82 \mathrm{C}$ & $96.01 \mathrm{~B}$ & $100.44 \mathrm{~A}$ & -8.58 & -4.41 \\
\hline $\begin{array}{c}\text { \% increase/ } \\
\text { decrease over }\end{array}$ & -8.58 & -4.41 & & & \\
\hline control & & & & & \\
\hline
\end{tabular}

*Treatment $1=$ Single irrigation. Treatment $2=$ Two irrigation, Treatment $3=$ Four irrigation

\section{Tillers plant ${ }^{-1}$}

The overall mean for tillers plant ${ }^{-1}$ of all wheat genotypes among three treatments showed significant difference $(\mathrm{P}<0.05)$. $\mathrm{T} 1$ showed significant decrease in tillers plant ${ }^{-}$ ${ }^{1}$ (5.67) as compared to T2 and T3 (6.08 and
6.39). Highly significant differences $(\mathrm{P}<0.05)$ for the trait tillers plant $^{-1}$ were recorded in wheat genotypes at $\mathrm{T} 1$ (Table 9). At T1, tillers plant ${ }^{-1}$ ranged from 3.80 in genotype Khirman to 7.13 in genotype BWDYT-4. Four test entries (BWDYT-1, 
BWDYT-7, BWDYT-9, DH3/25) and a check variety NIA-Amber showed significantly $(\mathrm{P}<0.05)$ increase in tillers plant $^{-1}$ at T1. Highly significant differences $(\mathrm{P}<0.05)$ for the trait tillers plant $^{-1}$ were recorded in wheat genotypes at $\mathrm{T} 2$ (Table 2). At $T 2$, tillers plant ${ }^{-1}$ ranged from 4.60 in genotype $\mathrm{DH} 8 / 13$ to 8.13 in genotype BWDYT-7. Two test entries (BWDYT-4 and BWDYT-6) and a check variety NIAAmber showed significant $\quad(\mathrm{P}<0.05)$ increase in tillers plant ${ }^{-1}$ at $\mathrm{T} 2$. Highly significant differences $(\mathrm{P}<0.05)$ for the trait tillers plant $^{-1}$ were recorded in wheat genotypes T3. At T3, tillers plant ${ }^{-1}$ ranged from 5.46 in genotype DH8/13 and Khirman to 8.20 in genotype BWDYT-2. Four test entries (BWDYT-4, BWDYT-5, BWDYT-6, BWDYT-7) and check variety NIA-Amber showed significant $(\mathrm{P}<0.05)$ increase in tillers plant ${ }^{-1}$ at $\mathrm{T} 3$.

Table 9. Overall mean performance for Tillers plant $^{-1}$ of wheat genotypes as affected by three different water stress conditions

\begin{tabular}{|c|c|c|c|c|c|}
\hline \multirow{2}{*}{ Genotypes } & \multicolumn{5}{|c|}{ Tillers plant ${ }^{-1}$} \\
\cline { 2 - 6 } & $\mathrm{T} 1$ & $\mathrm{~T} 2$ & $\mathrm{~T} 3$ & $\begin{array}{c}\text { T1\% } \\
\text { increase/ } \\
\text { decrease } \\
\text { over } \begin{array}{c}\text { control } \\
\mathrm{T} 3\end{array}\end{array}$ & $\begin{array}{c}\text { T2\% } \\
\text { increase/ } \\
\text { decrease } \\
\text { over control } \\
\mathrm{T} 3\end{array}$ \\
\hline BWDYT-1 & $6.00 \mathrm{ABCDE}$ & $6.06 \mathrm{BCD}$ & $6.13 \mathrm{BCD}$ & -2.12 & -1.14 \\
\hline BWDYT-2 & $5.66 \mathrm{BCDEF}$ & $5.06 \mathrm{CD}$ & $8.20 \mathrm{~A}$ & -30.98 & -38.29 \\
\hline BWDYT-3 & $6.40 \mathrm{BCD}$ & $5.66 \mathrm{BCD}$ & $6.13 \mathrm{BCD}$ & 4.40 & -7.67 \\
\hline BWDYT-4 & $7.13 \mathrm{~A}$ & $7.00 \mathrm{AB}$ & $7.00 \mathrm{ABCD}$ & 1.86 & 0.00 \\
\hline BWDYT-5 & $5.13 \mathrm{DEFG}$ & $5.60 \mathrm{BCD}$ & $6.86 \mathrm{ABCD}$ & -25.22 & -18.37 \\
\hline BWDYT-6 & $5.40 \mathrm{CDEF}$ & $6.66 \mathrm{AB}$ & $7.20 \mathrm{ABC}$ & -25.00 & -7.50 \\
\hline BWDYT-7 & $6.86 \mathrm{AB}$ & $8.13 \mathrm{~A}$ & $7.60 \mathrm{AB}$ & -9.74 & 6.97 \\
\hline BWDYT-8 & $5.60 \mathrm{BCDEF}$ & $5.93 \mathrm{BCD}$ & $6.00 \mathrm{BCD}$ & -6.67 & -1.17 \\
\hline BWDYT-9 & $6.53 \mathrm{ABC}$ & $6.40 \mathrm{BC}$ & $6.00 \mathrm{BCD}$ & 8.83 & 6.67 \\
\hline BWDYT-10 & $4.46 \mathrm{FG}$ & $5.86 \mathrm{BCD}$ & $6.40 \mathrm{BCD}$ & -30.31 & -8.44 \\
\hline BWDYT-11 & $5.73 \mathrm{BCDEF}$ & $5.66 \mathrm{BCD}$ & $5.93 \mathrm{BCD}$ & -3.37 & -4.55 \\
\hline MASR-18 & $4.80 \mathrm{EFG}$ & $5.60 \mathrm{BCD}$ & $5.73 \mathrm{CD}$ & -16.23 & -2.27 \\
\hline MASR-64 & $5.33 \mathrm{CDEF}$ & $5.73 \mathrm{BCD}$ & $5.73 \mathrm{CD}$ & -6.98 & 0.00 \\
\hline DH3/25 & $5.86 \mathrm{ABCDE}$ & $6.13 \mathrm{BC}$ & $6.13 \mathrm{BCD}$ & -4.40 & 0.00 \\
\hline DH8/13 & $5.20 \mathrm{CDEF}$ & $4.60 \mathrm{D}$ & $5.46 \mathrm{D}$ & -4.76 & -15.75 \\
\hline Khirman & $3.80 \mathrm{G}$ & $6.46 \mathrm{BC}$ & $5.46 \mathrm{D}$ & -30.40 & 18.32 \\
\hline NIA-Amber & $6.53 \mathrm{ABC}$ & $6.86 \mathrm{AB}$ & $6.66 \mathrm{ABCD}$ & -1.95 & 3.00 \\
\hline Overall mean & $5.67 \mathrm{~B}$ & $6.08 \mathrm{AB}$ & $6.39 \mathrm{~A}$ & -11.27 & -4.85 \\
\hline $\begin{array}{c}\text { \% increase/ } \\
\text { decrease over } \\
\text { control }\end{array}$ & -11.26 & -4.85 & & & \\
\hline
\end{tabular}

*Treatment $1=$ Single irrigation. Treatment $2=$ Two irrigation, Treatment $3=$ Four irrigation

\section{Spike length (cm)}

The overall mean for length of spike all wheat genotypes among three treatments showed significant difference $(\mathrm{P}<0.05)$. Non-significant difference was observed among overall mean of spike length of wheat genotypes. However, T2 and T3 $(11.32 \mathrm{~cm}$ and $11.44 \mathrm{~cm})$ showed comparatively increase in spike length then
T1 $(10.65 \mathrm{~cm})$. Highly significant differences $(\mathrm{P}<0.05)$ for the trait spike length were recorded in wheat genotypes at T1 (Table 10). At T1, spike length ranged from $8.80 \mathrm{~cm}$ in BWDYT-3 to $11.73 \mathrm{~cm}$ in genotype BWDYT-2 and Khirman. Five test entries (BWDYT-1, BWDYT-8, BWDYT-10, DH3/25 and DH8/13) showed significantly $(\mathrm{P}<0.05)$ increase in spike 
length at $\mathrm{T} 1$. a significant differences $(\mathrm{P}<0.05)$ for the trait spike length were recorded in wheat genotypes at T2 (Table 5). At $\mathrm{T} 2$, spike length ranged from $9.9 \mathrm{~cm}$ in genotype BWDYT-3 to $12.6 \mathrm{~cm}$ in genotype BWDYT-2. Two test entries (BWDYT-4 and BWDYT-5) showed significantly $(\mathrm{P}<0.05)$ increase in spike length at T2. Highly significant differences $(\mathrm{P}<0.05)$ for the trait Spike length were recorded in wheat genotypes at T3 (Table 5). Six test entries (BWDYT-4, BWDYT-7, BWDYT-8, BWDYT-11, MASR-18, DH8/13) showed significantly $(\mathrm{P}<0.05)$ increase in spike length at $\mathrm{T} 3$.

Table 10. Overall mean performance for Spike length $(\mathrm{cm})$ of wheat genotypes as affected by three different water stress conditions

\begin{tabular}{|c|c|c|c|c|c|}
\hline \multirow{2}{*}{ Genotypes } & \multicolumn{5}{|c|}{ Spike length (cm) } \\
\cline { 2 - 6 } & $\mathrm{T} 1$ & $\mathrm{~T} 2$ & $\mathrm{~T} 3$ & $\begin{array}{c}\text { \% increase/ } \\
\text { decrease in } \\
\text { T1 over } \\
\text { control (T3) }\end{array}$ & $\begin{array}{c}\text { \% increase/ } \\
\text { decrease in } \\
\text { T2 over } \\
\text { control (T3) }\end{array}$ \\
\hline BWDYT-1 & $11.61 \mathrm{AB}$ & $11.8 \mathrm{~B}$ & $11.33 \mathrm{DE}$ & 2.47 & 4.15 \\
\hline BWDYT-2 & $11.73 \mathrm{~A}$ & $12.6 \mathrm{~A}$ & $12.79 \mathrm{~A}$ & -8.29 & -1.49 \\
\hline BWDYT-3 & $8.80 \mathrm{~F}$ & $9.9 \mathrm{E}$ & $10.53 \mathrm{~F}$ & -16.43 & -5.98 \\
\hline BWDYT-4 & $10.83 \mathrm{BC}$ & $11.8 \mathrm{AB}$ & $11.73 \mathrm{BCD}$ & -7.67 & 0.60 \\
\hline BWDYT-5 & $10.63 \mathrm{CD}$ & $11.9 \mathrm{AB}$ & $11.30 \mathrm{DE}$ & -5.93 & 5.31 \\
\hline BWDYT-6 & $9.90 \mathrm{DE}$ & $10.8 \mathrm{CD}$ & $10.60 \mathrm{~F}$ & -6.60 & 1.89 \\
\hline BWDYT-7 & $10.36 \mathrm{CD}$ & $11.2 \mathrm{BCD}$ & $11.70 \mathrm{BCD}$ & -11.45 & -4.27 \\
\hline BWDYT-8 & $10.94 \mathrm{ABC}$ & $11.6 \mathrm{BC}$ & $11.63 \mathrm{BCD}$ & -5.93 & -0.26 \\
\hline BWDYT-9 & $10.50 \mathrm{CD}$ & $11.3 \mathrm{BCD}$ & $11.43 \mathrm{CDE}$ & -8.14 & -1.14 \\
\hline BWDYT-10 & $10.93 \mathrm{ABC}$ & $11.5 \mathrm{BC}$ & $11.50 \mathrm{CDE}$ & -4.96 & 0.00 \\
\hline BWDYT-11 & $10.60 \mathrm{CD}$ & $11.6 \mathrm{~B}$ & $12.16 \mathrm{~B}$ & -12.83 & -4.61 \\
\hline MASR-18 & $10.53 \mathrm{CD}$ & $10.7 \mathrm{CDE}$ & $11.63 \mathrm{BCD}$ & -9.46 & -8.00 \\
\hline MASR-64 & $9.25 \mathrm{EF}$ & $10.7 \mathrm{DE}$ & $10.53 \mathrm{~F}$ & -12.16 & 1.61 \\
\hline DH3/25 & $11.66 \mathrm{~A}$ & $11.2 \mathrm{BCD}$ & $11.23 \mathrm{DE}$ & 3.83 & -0.27 \\
\hline DH8/13 & $10.98 \mathrm{ABC}$ & $11.1 \mathrm{BCD}$ & $12.03 \mathrm{BC}$ & -8.73 & -7.73 \\
\hline Khirman & $11.73 \mathrm{~A}$ & $11.2 \mathrm{BCD}$ & $11.43 \mathrm{CDE}$ & 2.62 & -2.01 \\
\hline NIA-Amber & $10.16 \mathrm{CD}$ & $10.8 \mathrm{CD}$ & $10.90 \mathrm{EF}$ & -6.79 & -0.92 \\
\hline Overall mean & $10.65 \mathrm{~A}$ & $11.32 \mathrm{~A}$ & $11.44 \mathrm{~A}$ & -6.91 & -1.05 \\
\hline $\begin{array}{c}\text { \% increase/ } \\
\text { decrease over } \\
\text { control }\end{array}$ & -6.90 & -1.04 & & & - \\
\hline
\end{tabular}

*Treatment $1=$ Single irrigation. Treatment2=Two irrigation, Treatment $3=$ Four irrigation

\section{Grains spike ${ }^{-1}$}

The overall mean for grains spike $^{-1}$ of all wheat genotypes among three treatments showed significant difference $(\mathrm{P}<0.05)$. T3 showed significant increase in grains spike ${ }^{1}(95.82)$ as compared to T1 and T2 (63.11 and 68.32). Highly significant differences $(\mathrm{P}<0.05)$ for the trait grains spike $^{-1}$ were recorded in wheat genotypes at $\mathrm{T} 1$ (Table 11). At T1, grains spike ${ }^{-1}$ ranged from 52.2 in genotype BWDYT-10 to 74.2 in genotype BWDYT-10. Seven test entries (BWDYT-1, BWDYT-3, BWDYT-4,
BWDYT-5, BWDYT-7, BWDYT-8, V26 DH3/25) and check variety Khirman showed significant $(\mathrm{P}<0.05)$ increase in grains spike $^{-1}$ at $\mathrm{T} 1$. A significant differences was recorded $(\mathrm{P}<0.05)$ among wheat genotypes at T2 (Table 2). At T2, grains spike ${ }^{-1}$ ranged from 56.73 in genotype MASR-64 to 74.73 in genotype BWDYT-2. Six test entries (BWDYT-1, BWDYT-3, BWDYT-4, BWDYT-5, BWDYT-7, BWDYT-8) showed significant $(\mathrm{P}<0.05)$ increase in grains spike $^{-1}$ at T2. Highly significant differences 
$(\mathrm{P}<0.05)$ for the trait grains spike ${ }^{-1}$ were recorded in wheat genotypes at T3 (Table 2). At T3, grains spike ${ }^{-1}$ ranged from 60.86 to 76.80 in genotype in genotype BWDYT6 and DH8/13. Seven test entries
(BWDYT-2, BWDYT-3, BWDYT-4, BWDYT-5, BWDYT-7, BWDYT-8, MASR-18) and a check variety Khirman showed significantly $(\mathrm{P}<0.05)$ increase in grains spike ${ }^{-1}$ at $\mathrm{T} 3$.

Table 11. Overall mean performance for Grains spike ${ }^{-1}$ of wheat genotypes as affected by three different water stress conditions

\begin{tabular}{|c|c|c|c|c|c|}
\hline \multirow[b]{2}{*}{ Genotypes } & \multicolumn{5}{|c|}{ Grains spike $^{-1}$} \\
\hline & $\mathrm{T} 1$ & $\mathrm{~T} 2$ & $\mathrm{~T} 3$ & $\begin{array}{c}\text { T1\% } \\
\text { increase/ } \\
\text { decrease } \\
\text { over control } \\
\text { T3 }\end{array}$ & $\begin{array}{c}\mathrm{T} 2 \% \\
\text { increase/ } \\
\text { decrease } \\
\text { over control } \\
\mathrm{T} 3\end{array}$ \\
\hline BWDYT-1 & $65.8 \mathrm{ABCD}$ & $70.40 \mathrm{ABC}$ & $67.60 \mathrm{DEFG}$ & -2.66 & 4.14 \\
\hline BWDYT-2 & 61.6 BCDE & $74.73 \mathrm{~A}$ & 69.20 ABCDEF & -10.98 & 7.99 \\
\hline BWDYT-3 & $67.4 \mathrm{ABC}$ & $71.13 \mathrm{ABC}$ & $72.13 \mathrm{ABCDE}$ & -6.56 & -1.39 \\
\hline BWDYT-4 & $69.4 \mathrm{AB}$ & $71.20 \mathrm{AB}$ & $76.40 \mathrm{AB}$ & -9.16 & -6.81 \\
\hline BWDYT-5 & $67.2 \mathrm{ABC}$ & $68.40 \mathrm{ABCD}$ & 69.93 ABCDE & -3.90 & -2.19 \\
\hline BWDYT-6 & 59.6 CDEF & $64.86 \mathrm{BCDE}$ & $60.86 \mathrm{G}$ & -2.07 & 6.57 \\
\hline BWDYT-7 & $68.8 \mathrm{AB}$ & $70.46 \mathrm{ABC}$ & $75.06 \mathrm{ABCD}$ & -8.34 & -6.13 \\
\hline BWDYT-8 & $69.6 \mathrm{AB}$ & $70.66 \mathrm{ABC}$ & 72.46 ABCDE & -3.95 & -2.48 \\
\hline BWDYT-9 & 59.5 CDEF & 63.73 CDEF & $61.13 \mathrm{G}$ & -2.67 & 4.25 \\
\hline BWDYT-10 & $52.2 \mathrm{~F}$ & $65.06 \mathrm{BCDE}$ & $61.86 \mathrm{FG}$ & -15.62 & 5.17 \\
\hline BWDYT-11 & 59.3 CDEF & 65.46 BCDE & 67.60 DEFG & -12.28 & -3.17 \\
\hline MASR-18 & $57.8 \mathrm{DEF}$ & $57.13 \mathrm{~F}$ & $75.26 \mathrm{ABC}$ & -23.20 & -24.09 \\
\hline MASR-64 & 61.3 BCDE & $56.73 \mathrm{~F}$ & 68.80 BCDEF & -10.90 & -17.54 \\
\hline DH3/25 & $66.3 \mathrm{ABC}$ & $62.86 \mathrm{DEF}$ & $65.06 \mathrm{EFG}$ & 1.91 & -3.38 \\
\hline DH8/13 & $55.3 \mathrm{EF}$ & $60.53 \mathrm{EF}$ & $76.80 \mathrm{~A}$ & -27.99 & -21.18 \\
\hline Khirman & $74.2 \mathrm{~A}$ & $59.80 \mathrm{EF}$ & $70.80 \mathrm{ABCDE}$ & 4.80 & -15.54 \\
\hline NIA-Amber & $57.0 \mathrm{EF}$ & $61.73 \mathrm{DEF}$ & 67.80 CDEFG & -15.93 & -8.95 \\
\hline Overall mean & $63.11 \mathrm{C}$ & $65.58 \mathrm{~B}$ & $69.34 \mathrm{~A}$ & -8.98 & -5.42 \\
\hline $\begin{array}{c}\text { \% increase/ } \\
\text { decrease over } \\
\text { control }\end{array}$ & -8.98 & -5.42 & -- & & \\
\hline
\end{tabular}

*Treatment $1=$ Single irrigation. Treatment2=Two irrigation, Treatment $3=$ Four irrigation

\section{Main spike yield (g)}

The overall mean for main spike yield of all wheat genotypes among three treatments showed significant difference $(\mathrm{P}<0.05)$. T3 (2.62) showed significant increase all main spike yield as compared to T1 and T2 (2.42 and 2.52), respectively (Table 12).Highly significant differences $(\mathrm{P}<0.05)$ for the trait main spike yield were recorded in wheat genotypes T1 (Table 2). At T1, main spike yield ranged from 1.95 in genotype NIAAmber to 2.75 in genotype Khirman. Seven test entries (BWDYT-1, BWDYT-2, BWDYT-4, BWDYT-5, BWDYT-6, BWDYT-7, BWDYT-8) showed significant $(\mathrm{P}<0.05)$ increase in main spike yield at $\mathrm{T} 1$ as compared to all other entries and local checks. At T2, main spike yield ranged from 2.00 in genotype NIA-Amber to 2.94 in genotype BWDYT-2. Five test entries (BWDYT-1, BWDYT-4, BWDYT5, BWDYT-8, BWDYT-11) and check variety Khirman showed significant $(\mathrm{P}<0.05)$ increase in main spike yield at T2.Highly significant differences $(\mathrm{P}<0.05)$ for the trait main spike yield were recorded in wheat genotypes at T3. At T3, main spike yield ranged from 2.15 in genotype NIAAmber to 3.24 in genotype BWDYT-8. A 
test entry (BWDYT-7) showed significant $(\mathrm{P}<0.05)$ increase in main spike yield at $\mathrm{T} 3$.

Table 12. Overall mean performance for Main spike yield (g) of wheat genotypes as affected by three different water stress conditions

\begin{tabular}{|c|c|c|c|c|c|}
\hline \multirow{2}{*}{ Genotypes } & \multicolumn{5}{|c|}{ Main spike yield (g) } \\
\cline { 2 - 6 } & T1 & T2 & T3 & $\begin{array}{c}\text { T1\% } \\
\text { increase/ } \\
\text { decrease } \\
\text { over } \\
\text { control T3 }\end{array}$ & $\begin{array}{c}\text { T2\% } \\
\text { increase/ } \\
\text { decrease } \\
\text { over } \\
\text { control T3 }\end{array}$ \\
\hline BWDYT-1 & $2.45 \mathrm{ABCD}$ & $2.82 \mathrm{AB}$ & $2.52 \mathrm{CDE}$ & -2.78 & 11.90 \\
\hline BWDYT-2 & $2.51 \mathrm{ABCD}$ & $2.94 \mathrm{~A}$ & $2.78 \mathrm{BCD}$ & -9.71 & 5.76 \\
\hline BWDYT-3 & $2.37 \mathrm{CD}$ & $2.62 \mathrm{ABCDE}$ & $2.52 \mathrm{CDE}$ & -5.95 & 3.97 \\
\hline BWDYT-4 & $2.50 \mathrm{ABCD}$ & $2.76 \mathrm{ABC}$ & $2.57 \mathrm{BCDE}$ & -2.72 & 7.39 \\
\hline BWDYT-5 & $2.61 \mathrm{ABC}$ & $2.71 \mathrm{ABCD}$ & $2.59 \mathrm{BCDE}$ & 0.77 & 4.63 \\
\hline BWDYT-6 & $2.47 \mathrm{ABCD}$ & $2.39 \mathrm{DEFG}$ & $2.45 \mathrm{DEF}$ & 0.82 & -2.45 \\
\hline BWDYT-7 & $2.47 \mathrm{ABCD}$ & $2.56 \mathrm{BCDEF}$ & $2.92 \mathrm{AB}$ & -15.41 & -12.33 \\
\hline BWDYT-8 & $2.71 \mathrm{AB}$ & $2.86 \mathrm{AB}$ & $3.24 \mathrm{~A}$ & -16.36 & -11.73 \\
\hline BWDYT-9 & $2.28 \mathrm{D}$ & $2.43 \mathrm{CDEF}$ & $2.32 \mathrm{EF}$ & -1.72 & 4.74 \\
\hline BWDYT-10 & $2.40 \mathrm{CD}$ & $2.52 \mathrm{BCDEF}$ & $2.80 \mathrm{BCD}$ & -14.29 & -10.00 \\
\hline BWDYT-11 & $2.40 \mathrm{CD}$ & $2.66 \mathrm{ABCDE}$ & $2.78 \mathrm{BCD}$ & -13.67 & -4.32 \\
\hline MASR-18 & $2.32 \mathrm{CD}$ & $2.06 \mathrm{GH}$ & $2.33 \mathrm{EF}$ & -0.43 & -11.59 \\
\hline MASR-64 & $2.25 \mathrm{DE}$ & $2.32 \mathrm{EFGH}$ & $2.57 \mathrm{BCDE}$ & -12.45 & -9.73 \\
\hline DH3/25 & $2.42 \mathrm{BCD}$ & $2.22 \mathrm{FGH}$ & $2.45 \mathrm{DEF}$ & -1.22 & -9.39 \\
\hline DH8/13 & $2.35 \mathrm{CD}$ & $2.41 \mathrm{DEFG}$ & $2.78 \mathrm{BCD}$ & -15.47 & -13.31 \\
\hline Khirman & $2.75 \mathrm{~A}$ & $2.65 \mathrm{ABCDE}$ & $2.83 \mathrm{BC}$ & -2.83 & -6.36 \\
\hline NIA-Amber & $1.95 \mathrm{E}$ & $2.00 \mathrm{H}$ & $2.15 \mathrm{~F}$ & -9.30 & -6.98 \\
\hline Overall mean & $2.42 \mathrm{~B}$ & $2.52 \mathrm{AB}$ & $2.62 \mathrm{~A}$ & -7.63 & -3.82 \\
\hline $\begin{array}{c}\text { \% increase/ } \\
\text { decrease over } \\
\text { control }\end{array}$ & -7.63 & -3.81 & & & \\
\hline
\end{tabular}

*Treatment $1=$ Single irrigation. Treatment2=Two irrigation, Treatment $3=$ Four irrigation

Seed Index (1000-grain weight g)

Wheat genotypes among three treatments showed significant difference $(\mathrm{P}<0.05)$ for 1000-grain weight $(\mathrm{g})$ of all $\mathrm{T} 2$ and $\mathrm{T} 3$. Significant increase recorded in 1000-grain weight (g) as compared to $\mathrm{T} 3(39.59 \mathrm{~g})$ (Table 13). Highly significant differences $(\mathrm{P}<0.05)$ for the trait 1000 -grain weight $(\mathrm{g})$ were observed in wheat genotypes at $\mathrm{T} 1$, where weight $(\mathrm{g})$ ranged from $34.40 \mathrm{~g}$ to $46.74 \mathrm{~g}$ in genotype Khirman and BWDYT4. Twelve test entries (BWDYT-1, BWDYT-2, BWDYT-3, BWDYT-5, BWDYT-7, BWDYT-8, BWDYT-10, BWDYT-11, MASR-64, DH3/25, DH8/13) and both check varieties showed significantly $(\mathrm{P}<0.05)$ increase in 1000 grain weight $(\mathrm{g})$ at $\mathrm{T} 1$. Highly significant differences $(\mathrm{P}<0.05)$ for the trait 1000 -grain weight $(\mathrm{g})$ were recorded in wheat genotypes at T3. At T1, 1000-grain weight (g) ranged from $37.52 \mathrm{~g}$ in genotype BWDYT-9 to $51.12 \mathrm{~g}$ in BWDYT-4. Twelve test entries (BWDYT-1, BWDYT2, BWDYT-3, BWDYT-5, BWDYT-6, BWDYT-8, BWDYT-10, BWDYT-11, MASR-18, MASR-64, DH3/25, DH8/13) and a check variety showed significantly $(\mathrm{P}<0.05)$ increase for 1000 -grain weight $(\mathrm{g})$ at $\mathrm{T} 3$. 
Table 13. Overall mean performance for 1000-grain weight (g) of wheat genotypes as affected by three different water stress conditions

\begin{tabular}{|c|c|c|c|c|c|}
\hline \multirow[b]{2}{*}{ Genotypes } & \multicolumn{5}{|c|}{ 1000-grain weight (g) } \\
\hline & T1 & $\mathbf{T} 2$ & T3 & $\begin{array}{l}\text { T1\% increase/ } \\
\text { decrease over } \\
\text { control T3 }\end{array}$ & $\begin{array}{c}\text { T2\% increase/ } \\
\text { decrease over } \\
\text { control T3 }\end{array}$ \\
\hline BWDYT-1 & $37.27 \mathrm{AB}$ & $44.00 \mathrm{~A}$ & $46.27 \mathrm{ABC}$ & -19.45 & -4.91 \\
\hline BWDYT-2 & $43.30 \mathrm{AB}$ & $43.29 \mathrm{~A}$ & $42.84 \mathrm{ABC}$ & 1.07 & 1.05 \\
\hline BWDYT-3 & $40.99 \mathrm{AB}$ & $44.96 \mathrm{~A}$ & $49.64 \mathrm{AB}$ & -17.43 & -9.43 \\
\hline BWDYT-4 & $46.74 \mathrm{~A}$ & $42.39 \mathrm{~A}$ & $51.12 \mathrm{~A}$ & -8.57 & -17.08 \\
\hline BWDYT-5 & $38.72 \mathrm{AB}$ & $47.11 \mathrm{~A}$ & $40.93 \mathrm{ABC}$ & -5.40 & 15.10 \\
\hline BWDYT-6 & $38.82 \mathrm{AB}$ & $45.19 \mathrm{~A}$ & $46.48 \mathrm{ABC}$ & -16.48 & -2.78 \\
\hline BWDYT-7 & $38.56 \mathrm{AB}$ & $37.45 \mathrm{~A}$ & $38.50 \mathrm{BC}$ & 0.16 & -2.73 \\
\hline BWDYT-8 & $42.26 \mathrm{AB}$ & $40.89 \mathrm{~A}$ & $43.55 \mathrm{ABC}$ & -2.96 & -6.11 \\
\hline BWDYT-9 & $36.23 \mathrm{~B}$ & $40.33 \mathrm{~A}$ & $37.52 \mathrm{C}$ & -3.44 & 7.49 \\
\hline BWDYT-10 & $41.81 \mathrm{AB}$ & $42.15 \mathrm{~A}$ & $47.08 \mathrm{ABC}$ & -11.19 & -10.47 \\
\hline BWDYT-11 & $38.75 \mathrm{AB}$ & $43.11 \mathrm{~A}$ & $43.74 \mathrm{ABC}$ & -11.41 & -1.44 \\
\hline MASR-18 & $35.49 \mathrm{~B}$ & $39.45 \mathrm{~A}$ & 41.74 ABC & -14.97 & -5.49 \\
\hline MASR-64 & $42.74 \mathrm{AB}$ & $44.84 \mathrm{~A}$ & $47.42 \mathrm{ABC}$ & -9.87 & -5.44 \\
\hline DH3/25 & $39.61 \mathrm{AB}$ & $40.52 \mathrm{~A}$ & $39.43 \mathrm{ABC}$ & 0.46 & 2.76 \\
\hline DH8/13 & $37.66 \mathrm{AB}$ & $40.07 \mathrm{~A}$ & $42.43 \mathrm{ABC}$ & -11.24 & -5.56 \\
\hline Khirman & $34.40 \mathrm{~B}$ & $43.86 \mathrm{~A}$ & $41.91 \mathrm{ABC}$ & -17.92 & 4.65 \\
\hline NIA-Amber & $39.67 \mathrm{AB}$ & $41.95 \mathrm{~A}$ & $37.28 \mathrm{C}$ & 6.41 & 12.53 \\
\hline Overall mean & $39.59 \mathrm{~B}$ & $42.44 \mathrm{~A}$ & $43.40 \mathrm{~A}$ & -8.78 & -2.21 \\
\hline $\begin{array}{c}\% \text { increase/ } \\
\text { decrease over } \\
\text { control }\end{array}$ & -8.77 & -2.21 & -- & & \\
\hline
\end{tabular}

*Treatment $1=$ Single irrigation. Treatment $2=$ Two irrigation, Treatment $3=$ Four irrigation

Grain yield plot $^{-1}(\mathrm{~g})$

Mean for grain yield $\operatorname{plot}^{-1}(\mathrm{~g})$ of all wheat genotypes among three treatments showed significant difference $(\mathrm{P}<0.05)$. Highly significant differences $(\mathrm{P}<0.05)$ for the trait grain yield per plot $(\mathrm{g})$ were recorded in wheat genotypes at $\mathrm{T} 1$ where grain yield plot $^{-1}(\mathrm{~g})$ ranged from $434.0 \mathrm{~g}$ to $669.6 \mathrm{~g}$ in genotype DH3/25 and MASR-64 (Table 14). Highly significant differences $(\mathrm{P}<0.05)$ for the trait grain yield $\operatorname{plot}^{-1}(\mathrm{~g})$ were recorded in wheat genotypes at $\mathrm{T} 2$ where grain yield plot $^{-1}(\mathrm{~g})$ ranged from $454.0 \mathrm{~g}$ to 646.0g in genotype Khirman and BWDYT5. Thirteen test entries (BWDYT-1, BWDYT-2, BWDYT-3, BWDYT-4, BWDYT-6, BWDYT-8, BWDYT-9, BWDYT-10, BWDYT-11, MASR-64, $\mathrm{DH} 3 / 25, \mathrm{DH} 8 / 13$ ) and a check variety NIAAmber produced significantly $(\mathrm{P}<0.05)$ the highest grain yield $\operatorname{plot}^{-1}(\mathrm{~g})$ at T2. At T3, grain yield $\mathrm{plot}^{-1}(\mathrm{~g})$ ranged from $486.0 \mathrm{~g}$ to $676.6 \mathrm{~g}$ in genotype (Table 14) BWDYT-7 and DH8/13. Thirteen test entries (BWDYT-1, BWDYT-2, BWDYT-3, BWDYT-4, BWDYT-5, BWDYT-6, BWDYT-8, BWDYT-9, BWDYT-10, BWDYT-11, MASR-18, MASR-64, $\mathrm{DH} 3 / 25)$ and both check varieties produced significant $(\mathrm{P}<0.05)$ increase ingrain yield $\operatorname{plot}^{-1}(\mathrm{~g})$ at T3.

\section{Discussion}

The effects of water stress during various critical growth stages were determines on early growth vigor, early ground cover, plant height $(\mathrm{cm})$, spike length $(\mathrm{cm})$, spikelets spike $^{-1}$, grain spike ${ }^{-1}$, main spike yield (g), tillers plant ${ }^{-1}$, days to booting, days to heading, days to maturity, days to grain filling period, peduncle length $(\mathrm{cm})$, biological yield plot $^{-1}(\mathrm{~g})$, grain yield plot $^{-1}$ (g), harvest index (\%) and 1000-grain weight $(\mathrm{g})$.

Data recorded on various traits were statistically analyzed. The results indicated that the genotypes, treatments and genotype 
$\mathrm{x}$ treatments interactions were highly and significantly $(\mathrm{P} \leq 0.01)$ different among other for different yield associated traits; which indicated the genetic diversity among the genotype. Genotypes performed differently and variably with each under water stress conditions imposed at various growth stages. The visible effects of environments over genotypic performance for most of the traits were also observed. A trivial number of genotypes perform exclusively under sever water stress conditions while other could not compete.
Significant effects of water stress were observed on early growth vigor, plant height, number of spikelets spike ${ }^{-1}$, main spike yield, number of tillers plant ${ }^{-1}$, days to booting, days to heading, grain filling period and peduncle length, whereas, nonsignificant differences were observed in genotypic mean squares for the traits early ground cover, spike length, number of grains spike ${ }^{-1}$, days to maturity, biological yield $\operatorname{plot}^{-1}(\mathrm{~g})$, grain yield plot $^{-1}(\mathrm{~g})$, harvest index $(\%)$ and thousand grains weight $(\mathrm{g})$.

Table 14. Overall mean performance for grain yield plot $^{-1}$ (g) of wheat genotypes as affected by three different water stress conditions

\begin{tabular}{|c|c|c|c|c|c|}
\hline \multirow[b]{2}{*}{ Genotypes } & \multicolumn{5}{|c|}{ Grain yield plot $^{-1}(\mathrm{~g})$} \\
\hline & T1 & $\mathbf{T 2}$ & T3 & $\begin{array}{l}\text { T1\% increase/ } \\
\text { decrease over } \\
\text { control T3 }\end{array}$ & $\begin{array}{l}\text { T2\% increase/ } \\
\text { decrease over } \\
\text { control T3 }\end{array}$ \\
\hline BWDYT-1 & $640.0 \mathrm{AB}$ & $507.3 \mathrm{AB}$ & $596.6 \mathrm{AB}$ & 7.27 & -14.97 \\
\hline BWDYT-2 & $539.0 \mathrm{ABC}$ & $564.6 \mathrm{AB}$ & $593.6 \mathrm{AB}$ & -9.20 & -4.89 \\
\hline BWDYT-3 & $578.0 \mathrm{ABC}$ & $596.6 \mathrm{AB}$ & $646.0 \mathrm{AB}$ & -10.53 & -7.65 \\
\hline BWDYT-4 & $443.3 \mathrm{BC}$ & $593.6 \mathrm{AB}$ & $529.3 \mathrm{AB}$ & -16.25 & 12.15 \\
\hline BWDYT-5 & $450.3 \mathrm{BC}$ & $646.0 \mathrm{~A}$ & $517.6 \mathrm{AB}$ & -13.00 & 24.81 \\
\hline BWDYT-6 & $496.6 \mathrm{ABC}$ & $529.3 \mathrm{AB}$ & $568.6 \mathrm{AB}$ & -12.66 & -6.91 \\
\hline BWDYT-7 & $447.6 \mathrm{BC}$ & $517.6 \mathrm{AB}$ & $486.0 \mathrm{~B}$ & -7.90 & 6.50 \\
\hline BWDYT-8 & $564.6 \mathrm{ABC}$ & $568.6 \mathrm{AB}$ & $522.0 \mathrm{AB}$ & 8.16 & 8.93 \\
\hline BWDYT-9 & $503.0 \mathrm{ABC}$ & $486.0 \mathrm{AB}$ & $602.0 \mathrm{AB}$ & -16.45 & -19.27 \\
\hline BWDYT-10 & $508.3 \mathrm{ABC}$ & $522.0 \mathrm{AB}$ & $546.6 \mathrm{AB}$ & -7.01 & -4.50 \\
\hline BWDYT-11 & $554.6 \mathrm{ABC}$ & $602.0 \mathrm{AB}$ & $646.6 \mathrm{AB}$ & -14.23 & -6.90 \\
\hline MASR-18 & $537.3 \mathrm{ABC}$ & $463.3 \mathrm{~B}$ & $628.6 \mathrm{AB}$ & -14.52 & -26.30 \\
\hline MASR-64 & $669.6 \mathrm{~A}$ & $519.6 \mathrm{AB}$ & $535.3 \mathrm{AB}$ & 25.09 & -2.93 \\
\hline DH3/25 & $434.0 \mathrm{C}$ & $599.3 \mathrm{AB}$ & $580.6 \mathrm{AB}$ & -25.25 & 3.22 \\
\hline DH8/13 & $476.0 \mathrm{ABC}$ & $567.0 \mathrm{AB}$ & $676.6 \mathrm{~A}$ & -29.65 & -16.20 \\
\hline Khirman & $476.6 \mathrm{ABC}$ & $454.0 \mathrm{~B}$ & $538.0 \mathrm{AB}$ & -11.41 & -15.61 \\
\hline NIA-Amber & $511.0 \mathrm{ABC}$ & $556.6 \mathrm{AB}$ & $541.3 \mathrm{AB}$ & -5.60 & 2.83 \\
\hline Overall mean & $519.43 \mathrm{~B}$ & $546.71 \mathrm{~B}$ & $640.75 \mathrm{~A}$ & -18.93 & -14.68 \\
\hline $\begin{array}{c}\% \text { increase/ } \\
\text { decrease over } \\
\text { control }\end{array}$ & -18.93 & -14.67 & -- & & \\
\hline
\end{tabular}

*Treatment $1=$ Single irrigation. Treatment2=Two irrigation, Treatment $3=$ Four irrigation

\section{Phenological traits}

Plant breeders are interested in development of new genotypes, which takes fewer days for booting stage as the genotype could get more days after heading. The mean performance for days to booting of all wheat genotypes as compared all other genotypes eight test entries BWDYT-1 to 9, MASR-64 and a check variety NIA-Amber showed decrease in days to booting in T1 (single irrigation). Six test entries BWDYT-2, BWDYT-3, BWDYT-4, BWDYT-6, BWDYT-7, BWDYT-9 and a check variety NIA-Amber headed earlier under severe water stress (T1) as compared to other genotype; could be more tolerant. Mean value for days to maturity wheat genotypes BWDYT-2, 
BWDYT-3, BWDYT-4 and MASR-64 showed early maturity than other contesting genotypes at high water stress conditions (T1); could be more suitable under stress conditions.

\section{Days to booting}

Taking fewer days for booting stage would be a promising character. The mean performance for days to booting of all wheat genotypes BWDYT-1, BWDYT-3, BWDYT-4, BWDYT-5, BWDYT-6, BWDYT-7, BWDYT-9, MASR-64 and a check variety NIA-Amber showed decrease in days to booting in T1 (single irrigation). [13] Reported the importance of booting days under water stress studies conducted on 20 exotic wheat genotypes earlier.

\section{Days to heading}

Days to heading, is a phenological trait in estimation of plant performance under stress conditions. Six test entries BWDYT2, BWDYT-3, BWDYT-4, BWDYT-6, BWDYT-7, BWDYT-9 and a check variety NIA-Amber headed earlier under severe water stress (T1) as compared to other genotype; could be more tolerant. [14] Also noted the yield potential of wheat genotypes under drought condition. The various studies reported that the water stress imposed at different growth periods during booting, heading and early grain filling stage significantly reduces the grain yield.

\section{Days to maturity}

Days to maturity, is considered to be the main factor in achieving appropriate yields and the early maturing genotypes are supposed to be more suitable under stressed or harsh environments [15]. Such genotypes have capability to escape from severe water stress and mature earlier. Mean value for days to maturity among wheat genotypes BWDYT-2, BWDYT-3, BWDYT-4 and MASR-64 showed early maturity than other contesting genotypes at high water stress conditions (T1); could be more suitable under stress conditions.

\section{Days to grain filling period}

Grain filling period is an important factor in drought condition; the longer grain fill period contributes towards higher yields. Genotypes BWDYT-1, BWDYT-5, BWDYT-6, BWDYT-8, BWDYT-10, BWDYT-11, MASR-64, DH3/25, DH8/13 took more days to grain filling period at $\mathrm{T} 1$; could be considered as tolerant. The agromorphological characters in 36 durum wheat lines, Grain filling period, plant height, number of grains spike ${ }^{-1}$, number of spikelet spike ${ }^{-1}$, number of spike and 1000grain weight correlated significant and positively with yield [16].

\section{Morphological traits}

Certain genotypes showed less reduction in their plant height as compared to other genotypes at T1 (single irrigation). At T1, genotypes BWDYT-1, BWDYT-5, BWDYT-8, BWDYT-11, MASR-64, and DH8/13 showed significantly less reduction in plant height which indicated the tolerance of genotypes to water stress environment. With two irrigations (T2), two new genotypesBWDYT-11 and MASR-64showed less reduction in plant height. Wheat genotypes BWDYT-1, BWDYT-7, BWDYT-9, DH3/25) and a check variety NIA-Amber showed more number of tillers plant ${ }^{-1}$ at $\mathrm{T} 1$ (single irrigation). Five wheat genotypes BWDYT1, BWDYT-8, BWDYT-10, DH3/25 and DH8/13 showed less reduction in spike length at severe stress (T1); could be more tolerant to water stress conditions. The mean performance for grains spike $^{-1}$ of all wheat genotypes BWDYT-1, BWDYT-3, BWDYT-4, BWDYT-5, BWDYT-7, BWDYT-8, and DH3/25 along with check variety Khirman showed less reduction in number of grains spike $^{-1}$ at higher stress (T1). Wheat genotypes showed different response for the trait main spike yield at various water stress conditions. Genotypes BWDYT-1, BWDYT-2, BWDYT-4, BWDYT-5, BWDYT-6, BWDYT-7, BWDYT-8) showed increase in main spike yield at $\mathrm{T} 1$ as compared to all other entries and local checks; might be less affected due to water stress. The 1000-grain weight (g) of wheat genotypes BWDYT-1, BWDYT2, BWDYT-3, BWDYT-5, BWDYT-7, 
BWDYT-8, BWDYT-10, BWDYT-11, MASR-64, DH3/25, DH8/13) and both check varieties showed less reduction at $\mathrm{T} 1$; could be selected. Ten wheat genotypes BWDYT-1, BWDYT-2, BWDYT-3, BWDYT-6, BWDYT-8, BWDYT-9, BWDYT-10, BWDYT-11, MASR-18, DH8/13 and check varieties produced more grain yield plot $^{-1}$ at severe stress (T1); could be selected as tolerant and high yielding genotypes under harsh environments.

\section{Plant Height (cm)}

The visible effects of different water stresses were recorded on plant height of wheat genotypes. Various genotypes showed less reduction in their plant height as compared to other genotypes at $\mathrm{T} 1$ (single irrigation). At T1, genotypes BWDYT-1, BWDYT-5, BWDYT-8, BWDYT-11, MASR-64, and DH8/13 showed significantly reduced their height which indicated the tolerance of genotypes to water stress environment. Two time irrigated (T2), new genotypes BWDYT-11 and MASR-64 showed less reduction in plant height. The minimum and maximum reductions in cultivars for plant height categorized first group as drought tolerant and second as susceptible ones. According to [17] who noted that withholding irrigation at booting and at anthesis stages caused significant reduction in wheat plant height. Plant height directly or indirectly contributes towards grain yield, yet it could serve as an important indicator of waterstress tolerance via normal or retarded plant height.

\section{Tillers plant ${ }^{-1}$}

The trait number of tillers plant ${ }^{-1}$ is the main yield contributing trait. Wheat genotypes BWDYT-1, BWDYT-7, BWDYT-9, DH3/25) and a check variety NIA-Amber showed more number of tillers plant ${ }^{-1}$ at $\mathrm{T} 1$ (single irrigation). [18] Found the visible effects of water stresses on tiller number of wheat genotypes.

\section{Spike length $(\mathrm{cm})$}

Five wheat genotypes BWDYT-1, BWDYT-8, BWDYT-10, DH3/25 and DH8/13 showed less reduction in spike length at severe stress (T1); could be more tolerant to water stress conditions. The significant correlation between spike length and grain yield in stress condition and reported that the ability of swing nitrogen in spike and ability of retransforming of it to seed is effect. However, in our studies spike length of genotypes were affected due to water stress. Water stress at stages before anthesis can reduce number of kernels ear ${ }^{-1}$ [19].

\section{Grain spike ${ }^{-1}$}

The mean performance for grains spike ${ }^{-1}$ of all wheat genotypes BWDYT-1, BWDYT3, BWDYT-4, BWDYT-5, BWDYT-7, BWDYT-8, and DH3/25 along with check variety Khirman reduced number of grains per spike ${ }^{-1}$ at higher stress (T1). These findings suggested that these genotypes could be selected or further confirmed for drought tolerance studies. Drought stress before the anthesis in spring wheat also reduces the number of grains spike ${ }^{-1}[20]$.

\section{Main spike yield (g)}

Wheat genotypes showed different response for the trait main spike yield at various water stress conditions. Genotypes BWDYT-1, BWDYT-2, BWDYT-4, BWDYT-5, BWDYT-6, BWDYT-7, BWDYT-8) showed increase in main spike yield at $\mathrm{T} 1$ as compared to all other entries and local checks; might be less affected due to water stress. Similar findings were also reported that identifying the genotypes which have high further transfer ability in drought stress condition especially in grain filling stage provides the possibility to increase yield of grains without any increase in the amount of consumed water [21].

\section{0-grain weight (g)}

The seed index or weight of 1000-grain is considered to be the most important yield contributing trait in wheat and several other crops and it also serves as a good indicator of stress tolerance via weight of kernel. The 1000-grain weight $(\mathrm{g})$ of wheat genotypes BWDYT-1, BWDYT-2, BWDYT-3, BWDYT-5, BWDYT-7, BWDYT-8, BWDYT-10, BWDYT-11, MASR-64, 
$\mathrm{DH} 3 / 25, \mathrm{DH} 8 / 13)$ and both check varieties showed less reduction at $\mathrm{T} 1$; could be selected. The yield components like number of grains and size of grains were decreased under pre-anthesis water stress treatment in wheat [22].

\section{Grain yield plot $^{-1}(\mathrm{~g})$}

Grain yield is the dependent trait over output of yield components and the environment. Ten wheat genotypes BWDYT-1, BWDYT-2, BWDYT-3, BWDYT-6, BWDYT-8, BWDYT-9, BWDYT-10, BWDYT-11, MASR-18, DH8/13 and check varieties produced more grain yield plot ${ }^{-1}$ at severe stress (T1); could be selected as tolerant and high yielding genotypes under harsh environments. [23] Observed the significant correlation between spike length and grain yield in stress condition.

\section{Conclusion}

It is concluded that water stress significantly impact on yield and associated traits. The advanced genotypes BWDYT-1, BWDYT-2, BWDYT-3, BWDYT-6, BWDYT-8, BWDYT-9, BWDYT-10, BWDYT-11, MASR-18, MASR-64 and DH8/13 and both the standard/ controlled varieties Khirman and NIA-Amber produced significantly the highest grain yield than other contesting genotypes under sever water stress conditions (single irrigation). These genotypes showed genetic improvement in various yield traits as compared to high yielding and high tillering variety NIA-Amber and droughttolerant variety Khirman which were used as standard or check varieties. Therefore, our finding suggested that these genotypes had potential to grow under stress condition as compared when to other genotypes. Moreover, analyzed wheat varieties under water-stressed environments and screening through this study are selected as droughttolerant genotypes for future breeding to enhance yield in drought areas.

\section{Authors' contributions}

Conceived and designed the experiments: AY Siyal \& FK Siyal, Performed the experiments: AL Siyal, Analyzed the data:
AL Siyal \& T Jatt, Contributed materials/ analysis/ tools: AY Siyal \& FK Siyal, Wrote the paper: AL Siyal \& T Jatt.

\section{Acknowledgements}

The authors wished to thank Dr. Mahboob Ali Siyal, Deputy Chief Scientist, Nuclear Institute of Agriculture Tandojam for providing technical help and plant material such as local check varieties Amber and Khirman. We also thankful to International Maize and Wheat Improvement center (CIMMYT) Mexico and International Center for Agricultural Research in the Dry Areas, ICARDA, Lebanon for providing breeding lines of wheat.

\section{Reference}

1. Li H, Guo Q, Jing Y, Liu Z, Zheng Z, Sun Y, Xue Q \& Lai H (2019). Application of Streptomyces pactum Act12 enhances drought resistance in wheat. $J$ of Plant Growth Regul 39: 122-132.

2. Chiappero J, Del RCL, Alderete LGS, Palermo TB \& Banchio E (2019). Plant growth promoting rhizobacteria improve the antioxidant status in Menthapiperita grown under drought stress leading to an enhancement of plant growth and total phenolic content. Ind Crops Prod 139.

3. Agbodjato NA, Amogou OE, Noumavo PA, Dagbenonbakin G, Salami HA, Karimou R, Allad e A-M, Adebayo O, Baba-Moussa F \& Adjanohoun A (2018). Biofertilising, plant-stimulating and biocontrol potentials of maize plant growth promoting rhizobacteria isolated in central and northern Benin. Afr $J$ Microbiol Res 12: 664-672.

4. Toscano S, Ferrante A \& Romano D (2019). Response of mediterranean ornamental plants to drought stress. Horticulturae 5: 6.

5. Grzesiak S, Hordyńska N, Szczyrek P, Grzesiak MT, Noga A \& SzechyńskaHebda M (2019). Variation among wheat (Triticum easativum L.) genotypes in response to the drought stress: I selection approaches. J Plant Interact 14: 30-44.

6. Kumar S, Kumar P, and Kerkhi SA (2017). Genetic analysis for various yield components and gluten content in bread wheat (Triticumaestivum L.). J Appl Nat Sci 9(2): 879-882. 
7. Kour D, Rana KL, Yadav AN, Sheikh I, Kumar V, Dhaliwal HS \& Saxena AK (2020). Amelioration of drought stress in Foxtail millet (Setariaitalica L.) by P-solubilizing drought-tolerant microbes with multifarious plant growth promoting attributes. Soc for Environ Sust 3: 23-43

8. Rana KL, Kour D \& Yadav AN (2019). Endophytic microbiomes: biodiversity, ecological significance and biotechnological applications. Res $J$ Biotechnol 14: 142-162.

9. Danish S \& Zafar-ul-Hye M (2019). Coapplication of ACC-deaminase producing PGPR and timber-waste biochar improves pigments formation, growth and yield of wheat under drought stress. Sci Rep 9: 5999.

10. Rana KL, Kour D, Kaur T, Kour I, Shaikh AN, Yadav V, Kmuar A, Suman HS \& Dhaliwal (2020). Endophytic Microbes from Diverse Wheat Genotypes and Their Potential Biotechnological Applications in Plant Growth Promotion and Nutrient Uptake. Proc Natl Acad Sci, India, Sect B Biol Sci.

11. Johan A \& Ahmad F (2017). Effect of drought stress on growth and yield of wheat genotypes. Bangladesh Agron $J$ 20(2): 97-105

12. Yadav AN, Verma P, Kour D, Rana KL, Kumar V, Singh B, Chauahan VS, Sugitha T, Saxena AK \& Dhaliwal HS (2017). Plant microbiomes and its beneficial multifunctional plant growth promoting attributes. Intern J Environ Sci Nat Resour 3:1-8.

13. Ma J, Li R, Wang H, Li D, Wang X, Zhang Y, Zhen W, Duan H, Yan G \& Li Y (2017). Transcriptomics analyses reveal wheat responses to drought stress during reproductive stages under field conditions. Front Plant Sci 8: 592.

14. Vaishnav A \& Choudhary DK (2019). Regulation of drought-responsive gene expression in Glycine max 1. Merrill is mediated through Pseudomonas simiae strain AU. J Plant Growth Regul 38:333342

15. Chandio AA, Jiang Y, Joyo MA \& Rehman A (2016). Impact of area under cultivation, water availability, credit disbursement, and fertilizer off-take on wheat production in Pakistan. $J$ of Appl Enviro and Biol Sci 6(10): 10-18.

16. Siyal AL (2017). Effect of bio fertilizer in addition with phosphorus on the growth of maize (zeamayz L.). Intern $J$ of Adv Res 5 (12): 527-532.

17. Zhang X, Lei L, Lai J, Zhao H \& Song W (2018). Effects of drought stress and water recovery on physiological responses and gene expression in maize seedlings. $B M C$ Plant Biol 18: 68.

18. Kumari B, Mallick MA, Solanki MK, Solanki AC, Hora A \& Guo W (2019). Plant growth promoting rhizobacteria (PGPR): modern prospects for sustainable agriculture. In: Ansari RA, Mahmood I (eds) Plant health under biotic stress, vol 2. Microbial interactions. Springer Singapore, Singapore, pp. 109-127.

19. Zampieri M, Ceglar A, Dentener F, Toreti A (2017). Wheat yield loss attributable to heat waves, drought and water excess at the global, national and subnational scales. Environ Res Lett 12: 064008.

20. Barutcular C, Sabagh AEL, Koç M \& Ratnasekera D (2017). Relationships between grain yield and physiological traits of durum wheat varieties under drought and high temperature stress in Mediterranean conditions. Fresenius Environ Bull 26(4): 4282-4291.

21. Hossain MM, Hossain A, Alam M.A, Sabagh AEL, Khandakar FM, Haque MM, Muriruzzaman M, Islam MZ \& Das S (2018). Evaluation of fifty spring wheat genotypes grown under heat stress condition in multiple environments of Bangladesh. Fresenius Environ Bull 27(9): 5993-6004.

22. Devesh P, Moitra PK, Shukla RS \& Pandey S (2019). Genetic diversity and principal component analyses for yield, yield components and quality traits of advanced lines of Wheat. J Pharma Phytochem 8(3): 4834-4839.

23. Gao HM, Wang YF, Xu P \& Zhang ZB (2018). Overexpression of a WRKY transcription factor TaWRKY2 enhances drought stress tolerance in transgenic wheat. Front Plant Sci 9: 997. 